\title{
Variational properties of the first curve of the Fučík spectrum for elliptic operators
}

\author{
Riccardo MOLLE ${ }^{a}$, Donato PASSASEO ${ }^{b}$ \\ ${ }^{a}$ Dipartimento di Matematica, Università di Roma "Tor Vergata", \\ Via della Ricerca Scientifica n. 1, 00133 Roma, Italy. \\ ${ }^{b}$ Dipartimento di Matematica "E. De Giorgi", Università di Lecce, \\ P.O. Box 193, 73100 Lecce, Italy.
}

ABstract. - In this paper we present a new variational characteriztion of the first nontrival curve of the Fučík spectrum for elliptic operators with Dirichlet boundary conditions. Moreover, we describe the asymptotic behaviour and some properties of this curve and of the corresponding eigenfunctions. In particular, this new characterization allows us to compare the first curve of the Fučík spectrum with the infinitely many curves we obtained in previous works (see [36, 37]): for example, we show that these curves are all asymptotic to the same lines as the first curve, but they are all distinct from such a curve.

MSC: 35J20; 35J25; 35J61.

Keywords: Elliptic operators, Fučík spectrum, first curve.

\section{Introduction}

The Fučík spectrum, first introduced in [22] and [17], plays an important role in the study of some elliptic problems with linear growth. Let us consider, for example, the Dirichlet problem

$$
\Delta u+g(x, u)=0 \quad \text { in } \Omega, \quad u=0 \quad \text { on } \partial \Omega,
$$

where $\Omega$ is a bounded connected domain of $\mathbb{R}^{N}$ with $N \geq 1$ and $g$ is a Carathéodory function in $\Omega \times \mathbb{R}$ such that

$$
\lim _{t \rightarrow-\infty} \frac{g(x, t)}{t}=\alpha, \quad \lim _{t \rightarrow+\infty} \frac{g(x, t)}{t}=\beta \quad \forall x \in \Omega
$$

E-mail address: molle@mat.uniroma2.it (R. Molle). 
with $\alpha, \beta$ in $\mathbb{R}$. Existence and multiplicity of solutions for problems of this type are strictly related to the position of the pair $(\alpha, \beta)$ with respect to the Fučík spectrum $\Sigma$ which is defined as the set of all the pairs $(\alpha, \beta) \in \mathbb{R}^{2}$ such that the Dirichlet problem

$$
\Delta u-\alpha u^{-}+\beta u^{+}=0 \quad \text { in } \Omega, \quad u=0 \quad \text { on } \partial \Omega
$$

has nontrivial solutions (here, $u^{ \pm}=\max \{ \pm u, 0\}$ and $u$ nontrivial means $u \in H_{0}^{1}(\Omega)$, $u \not \equiv 0)$. In fact, these problems may lack compactness in the sense that the well known Palais-Smale compactness condition fails if the pair $(\alpha, \beta)$ belongs to the Fučík spectrum $\Sigma$; moreover, the topological properties of the sublevels of the corresponding energy functional depend on the position of $(\alpha, \beta)$ with respect to $\Sigma$.

After the pionering papers [10] and [1] on these problems, the important role of the Fučík spectrum has been pointed out in [22] and [17]. Then, several works have been devoted to describe the structure of $\Sigma$ (see, for example, [2, 5, 6, 8, 9, 16-30, 39-43] etc. ...).

Let us denote by $\lambda_{1}<\lambda_{2} \leq \lambda_{3} \leq \ldots$ the eigenvalues of $-\Delta$ in $H_{0}^{1}(\Omega)$. It is clear that $\Sigma$ includes the lines $\left\{\lambda_{1}\right\} \times \mathbb{R}$ and $\mathbb{R} \times\left\{\lambda_{1}\right\}$, contains all the pairs $\left(\lambda_{i}, \lambda_{i}\right) \forall i \in \mathbb{N}$ (that are the only pairs $(\alpha, \beta)$ of $\Sigma$ such that $\alpha=\beta)$ and is symmetric with respect to the line $\left\{(\alpha, \beta) \in \mathbb{R}^{2}: \alpha=\beta\right\}$ (because a function $u$ satisfies (1.3) if and only if $-u$ satisfies (1.3) with $(\beta, \alpha)$ in place of $(\alpha, \beta))$. Moreover, if $\alpha \neq \lambda_{1}, \beta \neq \lambda_{1}$ and $(\alpha, \beta) \in \Sigma$, then $\alpha>\lambda_{1}, \beta>\lambda_{1}$ and the eigenfunctions corresponding to the pair $(\alpha, \beta)$ are sign changing functions.

In the case $N=1, \Sigma$ is completely known and may be obtained by direct computation. It consists of curves emanating from the pairs $\left(\lambda_{i}, \lambda_{i}\right)$; if $i$ is an even positive integer, there exists only one curve while, if $i$ is odd, there exist exactly two curves emanating from $\left(\lambda_{i}, \lambda_{i}\right)$. All these curves are smooth, unbounded and decreasing. Moreover, on each curve, $\alpha$ tends to an eigenvalue of $-\Delta$ in $H_{0}^{1}(\Omega)$ as $\beta \rightarrow+\infty$. Conversely, for every eigenvalue $\lambda_{i}$ there exist exactly three curves asymptotic to the lines $\left\{\lambda_{i}\right\} \times \mathbb{R}$ and $\mathbb{R} \times\left\{\lambda_{i}\right\}$; they pass, respectively, through the pairs $\left(\lambda_{2 i-1}, \lambda_{2 i-1}\right),\left(\lambda_{2 i}, \lambda_{2 i}\right)$ and $\left(\lambda_{2 i+1}, \lambda_{2 i+1}\right)$.

On the contrary, in the case of higher dimensions the known results are much less complete and the description of $\Sigma$ remains a largely open question. It is known that $\Sigma$ is a closed set and that the lines $\left\{\lambda_{1}\right\} \times \mathbb{R}$ and $\mathbb{R} \times\left\{\lambda_{1}\right\}$ (the trivial part of $\Sigma$ ) are isolated in $\Sigma$ (see [17]). Many results concern the curves of $\Sigma$ emanating from each pair $\left(\lambda_{i}, \lambda_{i}\right)$ (local existence and multiplicity, local and global properties, etc. ...). In particular, if $\lambda_{i}$ has multiplicity $m$, i.e. $\lambda_{i-1}<\lambda_{i}=\ldots=\lambda_{i+m-1}<\lambda_{i+m}$, from the point $\left(\lambda_{i}, \lambda_{i}\right)$ arises a continuum composed by a lower and an upper curve, both decreasing (and maybe coincident) such that $\Sigma \cap\left(\lambda_{i-1}, \lambda_{i+m}\right)^{2}$ lies between these two curves, so the open squares $\left(\lambda_{i-1}, \lambda_{i}\right)^{2}$ and $\left(\lambda_{i}, \lambda_{i+m}\right)^{2}$ do not contain any point of $\Sigma$ (see, for example, $[24,29,41,42]$ and the references therein).

Combining these results, one can infer that $\Sigma$ contains a first nontrivial curve, which passes through $\left(\lambda_{2}, \lambda_{2}\right)$ and extends to infinity. In [20] the authors prove directly the 
existence of such a first curve, give a variational characterization of it and derive several properties. In particular, they show that this curve is asymptotic to the lines $\left\{\lambda_{1}\right\} \times \mathbb{R}$ and $\mathbb{R} \times\left\{\lambda_{1}\right\}$, give a new proof of the fact that these lines are isolated in $\Sigma$ and deduce that all the eigenfunctions corresponding to points of the first curve have exactly two nodal domains (extending the well known Courant nodal domains theorem).

Recently (see [33-35]) we have obtained new existence and multiplicity results for a class of Dirichlet problems of type (1.1) (in particular for semilinear problems with jumping nonlinearities) using a variational method that does not require to know whether or not the pair $(\alpha, \beta)$ belongs to $\Sigma$ and, in addition, may be used to give new information on the structure of $\Sigma$. In fact (see [36, 37]) using this method we have proved that if $N \geq 2$ there exist infinitely many curves in $\Sigma$, asymptotic to the lines $\left\{\lambda_{1}\right\} \times \mathbb{R}$ and $\mathbb{R} \times\left\{\lambda_{1}\right\}$ (while, if $N=1, \Sigma$ has only two curves asymptotic to these lines). More precisely (see also Theorem 3.1) we have proved that, if $N \geq 2$ and $k \in \mathbb{N}$, for $\beta>0$ large enough there exists $\alpha_{k, \beta}>\lambda_{1}$ such that $\left(\alpha_{k, \beta}, \beta\right) \in \Sigma$; moreover, for all $k \in \mathbb{N}$, $\lim _{\beta \rightarrow+\infty} \alpha_{k, \beta}=\lambda_{1}, \alpha_{k, \beta}$ depends continuously on $\beta$ and $\alpha_{k, \beta}<\alpha_{k+1, \beta}$ (notice that the method developed in [33-35] has been also used in the study of some nonlinear scalar field equations (see [11-13])).

The following natural question remains still open: where do these curves come from? Most probably, they come from bifurcations of the first nontrivial curve of $\Sigma$ or from pairs $\left(\lambda_{i}, \lambda_{i}\right)$ of higher eigenvalues.

The results obtained in [32] seem to confirm our conjecture. In fact, in [32] the authors study the Fučík spectrum of the laplacian on a two-dimensional torus $T^{2}$ with periodic conditions and, exploiting the invariance properties of $T^{2}$ with respect to translations, they prove that at least two global curves emanate from every pair of eigenvalues: a global curve which can be obtained explicitely and a global curve which can be characterized variationally using a suitable topological index (see $[4,7,31]$ ). The explicit curves are asymptotic to lines $\{\lambda\} \times \mathbb{R}$ and $\mathbb{R} \times\{\lambda\}$ for suitable constants $\lambda>0$, while the variational curves are all asymptotic to $\{0\} \times \mathbb{R}$ and $\mathbb{R} \times\{0\}$, the trivial lines of the Fučík spectrum in $T^{2}$. Therefore, the explicit and the variational curves cannot coincide globally and many curve crossings must occur. Moreover, on the first explicit curve there exist infinitely many points of bifurcations (associated to symmetry breaking phenomena).

These results suggest that, in higher dimensions, $\Sigma$ has a quite complicated structure even if, for example, $\Omega$ is a ball of $\mathbb{R}^{N}$ with $N \geq 2$ (where we observed similar phenomena that motivated our conjecture).

In the present paper we give a new variational characterization of the first nontrivial curve of $\Sigma$. We prove that the infimum

$$
\inf \left\{\int_{\Omega}\left|D u^{-}\right|^{2} d x: u \in H_{0}^{1}(\Omega),\left\|u^{+}\right\|_{L^{2}(\Omega)}=\left\|u^{-}\right\|_{L^{2}(\Omega)}=1, \int_{\Omega}\left|D u^{+}\right|^{2} d x=\beta\right\}
$$


is achieved for all $\beta>\lambda_{1}$; moreover we prove that, if we denote by $\alpha_{\beta}$ the above infimum, then $\alpha_{\beta}$ is continuous and strictly decreasing with respect to $\beta$ in $] \lambda_{1},+\infty$ [, $\lim _{\beta \rightarrow+\infty} \alpha_{\beta}=\lambda_{1}, \lim _{\beta \rightarrow \lambda_{1}} \alpha_{\beta}=+\infty,\left(\alpha_{\beta}, \beta\right) \in \Sigma \forall \beta>\lambda_{1}$ and

$$
\left\{(\alpha, \beta) \in \Sigma: \alpha>\lambda_{1}, \beta>\lambda_{1}\right\} \subseteq\left\{(\alpha, \beta) \in \mathbb{R}^{2}: \beta>\lambda_{1}, \alpha \geq \alpha_{\beta} \forall \beta>\lambda_{1}\right\} .
$$

Therefore (using $\beta$ as parameter) the first nontrivial curve of $\Sigma$ may be described as the set $\left\{(\alpha, \beta) \in \mathbb{R}^{2}: \beta>\lambda_{1}, \alpha=\alpha_{\beta} \forall \beta>\lambda_{1}\right\}$ (a similar description holds if we use $\alpha$ as parameter).

The eigenfunctions corresponding to $\left(\alpha_{\beta}, \beta\right)$ are suitable smooth functions $u_{\beta} \in H_{0}^{1}(\Omega)$, such that $u_{\beta}^{+} \not \equiv 0, u_{\beta}^{-} \not \equiv 0$ in $\Omega$ and the function $\bar{u}_{\beta}=-\left\|u_{\beta}^{-}\right\|_{L^{2}(\Omega)}^{-1} u_{\beta}^{-}+\left\|u_{\beta}^{+}\right\|_{L^{2}(\Omega)}^{-1} u_{\beta}^{+}$is a minimizing function for (1.4).

This variational characterization of the first nontrivial curve of $\Sigma$, which is different from the ones obtained in [20] and in [32], has been first announced in [38].

All the properties of the first curve and of the corresponding eigenfunctions may be easily deduced from this new characterization. In the present paper, in particular, we deduce that all the curves we obtained in [37] are distinct from the first curve. In fact, suitable asymptotic estimates of $\alpha_{\beta}$, as $\beta \rightarrow+\infty$, allow us to say that for every positive integer $k$ we have $\alpha_{\beta}<\alpha_{k, \beta}$ when $\beta$ is large enough.

The asymptotic behaviour of the eigenfunctions $u_{\beta}$ corresponding to $\left(\alpha_{\beta}, \beta\right)$, as $\beta \rightarrow$ $+\infty$, shows that the support of $u_{\beta}^{+}$is localized near the boundary of $\Omega$ for $\beta$ large enough and that $\left\|u_{\beta}\right\|_{L^{2}(\Omega)}^{-1} u_{\beta} \rightarrow-e_{1}$ in $\Omega$, where $e_{1}$ denotes the positive eigenfunction of $-\Delta$ in $H_{0}^{1}(\Omega)$, corresponding to $\lambda_{1}$ and normalized in $L^{2}(\Omega)$. On the contrary, the eigenfunctions $u_{k, \beta}$ corresponding to the pairs $\left(\alpha_{k, \beta}, \beta\right)$, for $\beta$ large enough, have the support of $u_{k, \beta}^{+}$localized near the maximum points of $e_{1}$ (see [36, 37] and also Theorem 3.1 and Proposition 3.2). This fact suggests that, arguing as in [37], it is possible to construct a new class of infinitely many curves of $\Sigma$, asymptotic to the lines $\left\{\lambda_{1}\right\} \times \mathbb{R}$ and $\mathbb{R} \times\left\{\lambda_{1}\right\}$, corresponding to eigenfunctions having an arbitrarily large number of bumps localized near the boundary of $\Omega$ (see Remark 3.5 for more details about this construction).

The method we use in this paper is completely variational. For all $\beta>\lambda_{1}$, the eigenfunction $u_{\beta}$ corresponding to the pair $\left(\alpha_{\beta}, \beta\right)$ is obtained as critical point of the functional $f(u)=\int_{\Omega}\left[|D u|^{2}-\beta\left(u^{+}\right)^{2}\right] d x$ constrained on the set $S=\left\{u \in H_{0}^{1}(\Omega)\right.$ : $\left.\int_{\Omega}\left(u^{-}\right)^{2} d x=1\right\}$ (here, $\alpha_{\beta}$ arises as the Lagrange multiplier with respect to the constraint $S$ ). In Section 2 we introduce also another functional $f_{\beta, \varepsilon}$, converging to $f_{\beta}$ as $\varepsilon \rightarrow 0$, which for all $\varepsilon>0$ presents more manageable variational properties with respect to $f_{\beta}$; thus we first find constrained critical points for $f_{\beta, \varepsilon}$ and then we let $\varepsilon \rightarrow 0$ in order to obtain the variational characterization of the first nontrivial curve of $\Sigma$ (see Theorem 2.1). In Section 3 we analyse the asymptotic behaviour, as $\beta \rightarrow+\infty$, of this curve and of the corresponding eigenfunctions, we compare this curve with the infinitely many curves obtained in [37] and we discuss some possible generalizations, forthcoming results on related questions, etc. ... 


\section{Variational characterization of the first curve of $\Sigma$}

The following theorem gives a variational characterization of the first nontrivial curve of the Fučík spectrum $\Sigma$.

Theorem 2.1 Let $\Omega$ be a bounded connected domain of $\mathbb{R}^{N}$ with $N \geq 1$. For all $\beta>\lambda_{1}$, let us set

$$
\alpha_{\beta}=\inf \left\{\int_{\Omega}\left|D u^{-}\right|^{2} d x: u \in H_{0}^{1}(\Omega),\left\|u^{+}\right\|_{L^{2}(\Omega)}=\left\|u^{-}\right\|_{L^{2}(\Omega)}=1, \int_{\Omega}\left|D u^{+}\right|^{2} d x=\beta\right\} .
$$

Then, $\alpha_{\beta}>\lambda_{1},\left(\alpha_{\beta}, \beta\right) \in \Sigma \forall \beta>\lambda_{1}$ and $\alpha_{\beta} \leq \alpha$ for every $\alpha>\lambda_{1}$ such that $(\alpha, \beta) \in \Sigma$. Moreover, $\alpha_{\beta}$ is continuous and strictly decreasing with respect to $\beta$ in ]$\lambda_{1},+\infty\left[, \alpha_{\lambda_{2}}=\lambda_{2}\right.$, the infimum in (2.1) is achieved $\forall \beta>\lambda_{1}$ and an eigenfunction corresponding to the pair $\left(\alpha_{\beta}, \beta\right)$ is given by $u_{\beta}=-\bar{u}_{\beta}^{-}+\mu_{\beta} \bar{u}_{\beta}^{+}$, where $\bar{u}_{\beta}$ is a minimizing function for (2.1) and $\mu_{\beta}$ is a suitable positive constant.

As $\beta \rightarrow+\infty, \alpha_{\beta} \rightarrow \lambda_{1}$ and $u_{\beta}^{-} \rightarrow e_{1}$ in $H_{0}^{1}(\Omega) ;$ as $\beta \rightarrow \lambda_{1}, \alpha_{\beta} \rightarrow+\infty$ and $\left\|u_{\beta}^{+}\right\|_{L^{2}(\Omega)}^{-1} u_{\beta}^{+} \rightarrow e_{1}$ in $H_{0}^{1}(\Omega)$.

In order to prove this theorem, for all $\beta>0$ and $\varepsilon>0$ we consider the functional $f_{\beta, \varepsilon}: H_{0}^{1}(\Omega) \rightarrow \mathbb{R}$ defined by

$$
f_{\beta, \varepsilon}(u)=\int_{\Omega}|D u|^{2} d x-2 \int_{\Omega} G_{\beta, \varepsilon}(u) d x
$$

where $G_{\beta, \varepsilon}(t)=\int_{0}^{t} g_{\beta, \varepsilon}(\tau) d \tau \forall t \in \mathbb{R}$, with $g_{\beta, \varepsilon}(\tau)=0 \forall \tau \leq \varepsilon$ and $g_{\beta, \varepsilon}(\tau)=\beta(\tau-$

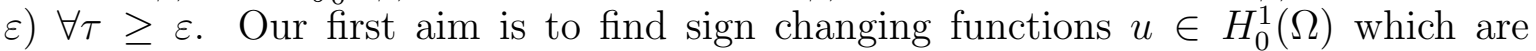
constrained critical points for the functional $f_{\beta, \varepsilon}$ constrained on the set $S=\left\{u \in H_{0}^{1}(\Omega)\right.$ : $\left.\int_{\Omega}\left(u^{-}\right)^{2} d x=1\right\}$. Therefore, we consider the set $M^{\beta, \varepsilon}$ consisting of all the functions $u$ in $S$ such that $u^{+} \not \equiv 0$ and $f_{\beta, \varepsilon}^{\prime}(u)\left[u^{+}\right]=0$.

One can easily verify that for all $\varepsilon>0$, if a sign changing function $u \in S$ is a critical point for $f_{\beta, \varepsilon}$ constrained on $S$, then $u \in M^{\beta, \varepsilon}$ and $f_{\beta, \varepsilon}^{\prime}\left(-u^{-}+t u^{+}\right)\left[u^{+}\right]$is positive for $t \in] 0,1\left[\right.$ and negative for $t>1$ (because $\frac{1}{\tau} g(\tau)$ is strictly increasing with respect to $\tau$ in ]$\varepsilon,+\infty[)$; so the function $u$ is the unique maximum point for $f_{\beta, \varepsilon}$ on the set $\left\{-u^{-}+t u^{+}\right.$ $: t \geq 0\}$ which is included in $S$.

Notice that, for $\varepsilon=0$, the functional $f_{\beta}=f_{\beta, 0}$ and the sets $M^{\beta, 0}$ do not have the same properties; this is the reason for introducing first the functional $f_{\beta, \varepsilon}$ which, for all $\varepsilon>0$, presents more manageable variational properties and then we let $\varepsilon \rightarrow 0$. 
Proposition 2.2 For all $\varepsilon>0$, we have $M^{\beta, \varepsilon} \neq \emptyset$ if and only if $\beta>\lambda_{1}$. If $\beta>\lambda_{1}$, the minimum of $f_{\beta, \varepsilon}$ on $M^{\beta, \varepsilon}$ is achieved for all $\varepsilon>0$ and every minimizing function $u_{\beta, \varepsilon}$ satisfies the equation

$$
\Delta u_{\beta, \varepsilon}+g_{\beta, \varepsilon}\left(u_{\beta, \varepsilon}\right)-\alpha_{\beta, \varepsilon} u_{\beta, \varepsilon}^{-}=0 \quad \text { in } \Omega,
$$

where $\alpha_{\beta, \varepsilon}=\int_{\Omega}\left|D u_{\beta, \varepsilon}^{-}\right|^{2} d x \forall \varepsilon>0, \forall \beta>\lambda_{1}$.

Proof Notice that $g_{\beta, \varepsilon}(\tau) \leq \beta \tau \forall \tau \geq 0, \forall \beta>0, \forall \varepsilon>0$; moreover, if a function $u \in S$ satisfies $\int_{\Omega}\left(u^{+}\right)^{2} d x>0$, then $\int_{\Omega}\left|D u^{+}\right|^{2} d x>\lambda_{1} \int_{\Omega}\left(u^{+}\right)^{2} d x$. Thus, if $\beta \leq \lambda_{1}$ we obtain $\int_{\Omega}\left|D u^{+}\right|^{2} d x>\beta \int_{\Omega}\left(u^{+}\right)^{2} d x \geq \int_{\Omega} g_{\beta, \varepsilon}\left(u^{+}\right) u^{+} d x$, namely $f_{\beta, \varepsilon}^{\prime}(u)\left[u^{+}\right]>0$, which implies $u \notin M^{\beta, \varepsilon}$. Therefore, if $\beta \leq \lambda_{1}$, we have $M^{\beta, \varepsilon}=\emptyset \forall \varepsilon>0$.

On the contrary, if $\beta>\lambda_{1}$ we have $M^{\beta, \varepsilon} \neq \emptyset$. In fact, if $\beta>\lambda_{1}$, one can easily construct a function $\bar{u} \in S$ such that $\int_{\Omega}\left|D \bar{u}^{+}\right|^{2} d x<\beta \int_{\Omega}\left(\bar{u}^{+}\right)^{2} d x$. For example, fix $x_{0} \in \partial \Omega$, set $\Omega_{r}=\Omega \backslash \bar{B}\left(x_{0}, r\right), \tilde{\Omega}_{r}=\Omega \cap B\left(x_{0}, r\right)$ and, for $\left.r \in\right] 0,(1 / 2) \operatorname{diam}(\Omega)[$, consider the positive eigenfunctions $e_{1}\left(\Omega_{r}\right)$ and $e_{1}\left(\tilde{\Omega}_{r}\right)$, normalized in $L^{2}$, corresponding to the first eigenvalues of the operator $-\Delta$ in $H_{0}^{1}\left(\Omega_{r}\right)$ and $H_{0}^{1}\left(\tilde{\Omega}_{r}\right)$ respectively; then, the function $\bar{u}$ such that $\bar{u}=e_{1}\left(\Omega_{r}\right)$ in $\Omega_{r}$ and $\bar{u}=-e_{1}\left(\tilde{\Omega}_{r}\right)$ in $\tilde{\Omega}_{r}$ has the required properties for $r$ small enough.

Notice that $f_{\beta, \varepsilon}\left(-\bar{u}^{-}+t \bar{u}^{+}\right)=f_{\beta, \varepsilon}\left(-\bar{u}^{-}\right)+f_{\beta, \varepsilon}\left(t \bar{u}^{+}\right) \forall t \geq 0$; moreover, one can easily verify by direct computation that

$$
\lim _{t \rightarrow+\infty} \frac{1}{t^{2}} f_{\beta, \varepsilon}\left(t \bar{u}^{+}\right)=\int_{\Omega}\left|D \bar{u}^{+}\right|^{2} d x-\beta \int_{\Omega}\left(\bar{u}^{+}\right)^{2} d x<0
$$

and (since $\varepsilon>0$ )

$$
\lim _{t \rightarrow 0} \frac{1}{t^{2}} f_{\beta, \varepsilon}\left(t \bar{u}^{+}\right)=\int_{\Omega}\left|D \bar{u}^{+}\right|^{2} d x>0 .
$$

Therefore, we infer that for all $\varepsilon>0$ there exists $\bar{t}>0$ such that $f_{\beta, \varepsilon}\left(-\bar{u}^{-}+\bar{t} \bar{u}^{+}\right) \geq$ $f_{\beta, \varepsilon}\left(-\bar{u}^{-}+t \bar{u}^{+}\right) \forall t \geq 0$, which implies $-\bar{u}^{-}+\bar{t} \bar{u}^{+} \in M^{\beta, \varepsilon}$. Thus, $M^{\beta, \varepsilon} \neq \emptyset \forall \beta>\lambda_{1}$, $\forall \varepsilon>0$.

Now, let us prove that the infimum $\inf _{M^{\beta, \varepsilon}} f_{\beta, \varepsilon}$ is achieved for all $\beta>\lambda_{1}$ and $\varepsilon>0$. Let us consider a minimizing sequence $\left(u_{n}\right)_{n}$. Notice that $f_{\beta, \varepsilon}\left(u_{n}\right)=f_{\beta, \varepsilon}\left(-u_{n}^{-}\right)+$ $f_{\beta, \varepsilon}\left(u_{n}^{+}\right) \forall n \in \mathbb{N}$, where $=f_{\beta, \varepsilon}\left(-u_{n}^{-}\right)=\int_{\Omega}\left|D u_{n}^{-}\right|^{2} d x \geq \lambda_{1}$ (because $\left\|u_{n}^{-}\right\|_{L^{2}(\Omega)}=1$ ) and $f_{\beta, \varepsilon}\left(u_{n}^{+}\right)>0$ since $u_{n} \in M^{\beta, \varepsilon}$ implies $f_{\beta, \varepsilon}\left(u_{n}^{+}\right)=\max \left\{f_{\beta, \varepsilon}\left(t u_{n}^{+}\right): t \geq 0\right\}>0$ $\forall \varepsilon>0$ (because $\frac{1}{\tau} g_{\beta, \varepsilon}(\tau)$ is strictly increasing with respect to $\tau$ in $] \varepsilon,+\infty[$ ). Taking into account that $\sup \left\{f_{\beta, \varepsilon}\left(u_{n}\right): n \in \mathbb{N}\right\}<+\infty$, it follows that

$$
\lambda_{1} \leq \liminf _{n \rightarrow \infty} f_{\beta, \varepsilon}\left(-u_{n}^{-}\right) \leq \limsup _{n \rightarrow \infty} f_{\beta, \varepsilon}\left(-u_{n}^{-}\right)<+\infty
$$

and

$$
0 \leq \liminf _{n \rightarrow \infty} f_{\beta, \varepsilon}\left(u_{n}^{+}\right) \leq \limsup _{n \rightarrow \infty} f_{\beta, \varepsilon}\left(u_{n}^{+}\right)<+\infty .
$$


Since $f_{\beta, \varepsilon}\left(-u_{n}^{-}\right)=\int_{\Omega}\left|D u_{n}^{-}\right|^{2} d x$, (2.6) implies that the sequence $\left(u_{n}^{-}\right)_{n}$ is bounded in $H_{0}^{1}(\Omega)$. Now, let us prove that also the sequence $\left(u_{n}^{+}\right)_{n}$ is bounded in $H_{0}^{1}(\Omega)$. Taking into account that $f_{\beta, \varepsilon}^{\prime}\left(u_{n}\right)\left[u_{n}^{+}\right]=0 \forall n \in \mathbb{N}$, we have

$$
\int_{\Omega}\left|D u_{n}^{+}\right|^{2} d x=\int_{\Omega} g_{\beta, \varepsilon}\left(u_{n}^{+}\right) u_{n}^{+} d x \leq \beta \int_{\Omega}\left(u_{n}^{+}\right)^{2} d x .
$$

Therefore, it suffices to prove that the sequence $\left(u_{n}^{+}\right)_{n}$ is bounded in $L^{2}(\Omega)$. Arguing by contradiction, assume that (up to a subsequence) $\lim _{n \rightarrow \infty} \int_{\Omega}\left(u_{n}^{+}\right)^{2} d x=\infty$ and set $v_{n}=\left\|u_{n}^{+}\right\|_{L^{2}(\Omega)}^{-1} u_{n}^{+}$. Then, (2.8) implies $\int_{\Omega}\left|D v_{n}\right|^{2} d x \leq \beta \forall n \in \mathbb{N}$. So (up to a subsequence) $\left(v_{n}\right)_{n}$ converges in $L^{2}(\Omega)$, weakly in $H_{0}^{1}(\Omega)$ and a.e. in $\Omega$ to a function $v \in H_{0}^{1}(\Omega)$. It follows that $\int_{\Omega}|D v|^{2} d x \leq \beta, \int_{\Omega} v^{2} d x=1$ and $v \geq 0$ in $\Omega$. Moreover, taking into account that $f_{\beta, \varepsilon}^{\prime}\left(u_{n}\right)\left[u_{n}^{+}\right]=0 \forall n \in \mathbb{N}$, and that $\lim _{n \rightarrow \infty} \int_{\Omega}\left(u_{n}^{+}\right)^{2} d x=\infty$, one can verify by direct computation that $\lim _{n \rightarrow \infty} \int_{\Omega}\left|D v_{n}\right|^{2} d x=\beta$. It follows that

$$
\lim _{n \rightarrow \infty} f_{\beta, \varepsilon}^{\prime}\left(t v_{n}\right)\left[v_{n}\right]=2 t \beta-2 \int_{\Omega} g_{\beta, \varepsilon}(t v) v d x \quad \forall t \geq 0 .
$$

Since $\int_{\Omega} v^{2} d x=1$, for all $\varepsilon>0$ we obtain

$$
\liminf _{t \rightarrow+\infty}\left[t \beta-\int_{\Omega} g_{\beta, \varepsilon}(t v) v d x\right]=\liminf _{t \rightarrow+\infty} \int_{\Omega}\left[\beta t v-g_{\beta, \varepsilon}(t v)\right] v d x>0 .
$$

Now, let us set $t_{n}=\left\|u_{n}^{+}\right\|_{L^{2}(\Omega)}$ and notice that $\left.f_{\beta, \varepsilon}^{\prime}\left(t v_{n}\right)\left[v_{n}\right]>0 \forall t \in\right] 0, t_{n}$ [ (because $\frac{1}{\tau} g_{\beta, \varepsilon}(\tau)$ is strictly increasing with respect to $\tau$ in $] \varepsilon,+\infty[)$. Since we are assuming $\lim _{n \rightarrow \infty} t_{n}=+\infty$, we obtain

$$
\liminf _{n \rightarrow \infty} f_{\beta, \varepsilon}\left(u_{n}^{+}\right)=\liminf _{n \rightarrow \infty} \int_{0}^{t_{n}} f_{\beta, \varepsilon}^{\prime}\left(t v_{n}\right)\left[v_{n}\right] d t \geq 2 \int_{0}^{\tau}\left[t \beta-\int_{\Omega} g_{\beta, \varepsilon}(t v) v d x\right] d t \quad \forall \tau>0 .
$$

Then, as $\tau \rightarrow+\infty$, from (2.10) we obtain $\lim _{n \rightarrow \infty} f_{\beta, \varepsilon}\left(u_{n}^{+}\right)=+\infty$, in contradiction with (2.7). Therefore, we can say that also the sequence $\left(u_{n}^{+}\right)_{n}$ is bounded in $H_{0}^{1}(\Omega)$. It follows that there exists $u \in H_{0}^{1}(\Omega)$ such that (up to a subsequence) $\left(u_{n}\right)_{n}$ converges to $u$ in $L^{2}(\Omega)$, weakly in $H_{0}^{1}(\Omega)$ and a.e. in $\Omega$. As a consequence of the $L^{2}(\Omega)$ convergence, we have $\int_{\Omega}\left(u^{-}\right)^{2} d x=1$. Let us prove that $u^{+} \not \equiv 0$. Arguing by contradiction, assume that $u^{+} \equiv 0$. Then (because of the $L^{2}(\Omega)$ convergence) from (2.8) we infer that $\lim _{n \rightarrow \infty} \int_{\Omega}\left|D u_{n}^{+}\right|^{2} d x=0$, so we have $\lim _{n \rightarrow \infty} f_{\beta, \varepsilon}\left(u_{n}^{+}\right)=0$. Therefore, we obtain a contradiction if we prove that

$$
\inf \left\{f_{\beta, \varepsilon}\left(w^{+}\right): w \in M^{\beta, \varepsilon}\right\}>0 \quad \forall \varepsilon>0 .
$$

Since $w \in M^{\beta, \varepsilon}$ implies $f_{\beta, \varepsilon}\left(w^{+}\right)=\max \left\{f_{\beta, \varepsilon}\left(t w^{+}\right): t>0\right\}$, it is clear that it suffices to prove that there exist two positive constants $\rho_{\beta, \varepsilon}$ and $c_{\beta, \varepsilon}$ such that $f_{\beta, \varepsilon}(w) \geq c_{\beta, \varepsilon}$ $\forall w \in H_{0}^{1}(\Omega)$ such that $\int_{\Omega}|D w|^{2} d x=\rho_{\beta, \varepsilon}$. 
In order to prove the existence of these constants $c_{\beta, \varepsilon}$ and $\rho_{\beta, \varepsilon}$, notice that, since $\beta>\lambda_{1}$, there exists $j_{\beta} \in \mathbb{N}$ such that $\lambda_{j_{\beta}} \leq \beta<\lambda_{j_{\beta}+1}$. Let us denote by $S_{\beta}^{1}$ and $S_{\beta}^{2}$ the closed subspaces of $H_{0}^{1}(\Omega)$ spanned by the eigenfunctions of the Laplace operator $-\Delta$ in $H_{0}^{1}(\Omega)$, corresponding to eigenvalues $\lambda_{j}$ with, respectively, $1 \leq j \leq j_{\beta}$ and $j \geq j_{\beta}+1$. For all $\beta>\lambda_{1}$ and $\varepsilon>0$, there exists $\nu_{\beta, \varepsilon}>0$ such that, if $w \in S_{\beta}^{1}$ and $\int_{\Omega}|D w|^{2} d x \leq \nu_{\beta, \varepsilon}^{2}$, then $|w(x)| \leq \varepsilon \forall x \in \Omega$.

For all $w \in H_{0}^{1}(\Omega)$ such that $\int_{\Omega}|D w|^{2} d x \leq \nu_{\beta, \varepsilon}^{2}$, set $w=w_{1, \beta}+w_{2, \beta}$, with $w_{1, \beta} \in S_{\beta}^{1}$ and $w_{2, \beta} \in S_{\beta}^{2}$. Then, taking into account that $\int_{\Omega}^{\beta}\left|D w_{1, \beta}\right|^{2} d x \leq \nu_{\beta, \varepsilon}^{2}$ and as a consequence $w_{1, \beta} \leq \varepsilon$ in $\Omega$, we have $f_{\beta, \varepsilon}\left(w_{1, \beta}\right)=\int_{\Omega}\left|D w_{1, \beta}\right|^{2} d x$ and $f_{\beta, \varepsilon}^{\prime}\left(w_{1, \beta}\right)\left[w_{2, \beta}\right]=0$. Therefore, we obtain

$$
f_{\beta, \varepsilon}(w)=f_{\beta, \varepsilon}\left(w_{1, \beta}+w_{2, \beta}\right)=f_{\beta, \varepsilon}\left(w_{1, \beta}+w_{2, \beta}\right)-f_{\beta, \varepsilon}\left(w_{1, \beta}\right)+\int_{\Omega}\left|D w_{1, \beta}\right|^{2} d x,
$$

where

$$
\begin{aligned}
f_{\beta, \varepsilon}\left(w_{1, \beta}+w_{2, \beta}\right)-f_{\beta, \varepsilon}\left(w_{1, \beta}\right) & \geq f_{\beta, \varepsilon}^{\prime}\left(w_{1, \beta}\right)\left[w_{2, \beta}\right]+\int_{\Omega}\left|D w_{2, \beta}\right|^{2} d x-\beta \int_{\Omega} w_{2, \beta}^{2} d x \\
& =\int_{\Omega}\left[\left|D w_{2, \beta}\right|^{2}-\beta w_{2, \beta}^{2}\right] d x \\
& \geq\left(1-\frac{\beta}{\lambda_{j_{\beta}+1}}\right) \int_{\Omega}\left|D w_{2, \beta}\right|^{2} d x
\end{aligned}
$$

because $\int_{\Omega}\left|D w_{2, \beta}\right|^{2} d x \geq \lambda_{j_{\beta}+1} \int_{\Omega} w_{2, \beta}^{2} d x$. It follows that, for a suitable $\tilde{c}_{\beta, \varepsilon}>0$, we have $f_{\beta, \varepsilon}(w) \geq \tilde{c}_{\beta, \varepsilon} \int_{\Omega}|D w|^{2} d x \forall w \in H_{0}^{1}(\Omega)$ such that $\int_{\Omega}|D w|^{2} d x \leq \nu_{\beta, \varepsilon}^{2}$. Therefore, it follows easily that there exist two constants $\left.\rho_{\beta, \varepsilon} \in\right] 0, \nu_{\beta, \varepsilon}\left[\right.$ and $c_{\beta, \varepsilon}>0$ satisfying the required properties. Thus, we can say that $u^{+} \not \equiv 0$.

From the weak $H_{0}^{1}(\Omega)$ convergence, it follows that $f_{\beta, \varepsilon}^{\prime}\left(u^{+}\right)\left[u^{+}\right] \leq 0$; on the other hand, a direct computation shows that $\lim _{t \rightarrow 0} \frac{1}{t} f_{\beta, \varepsilon}^{\prime}\left(t u^{+}\right)\left[u^{+}\right]=2 \int_{\Omega}\left|D u^{+}\right|^{2} d x$, so we infer that $f_{\beta, \varepsilon}^{\prime}\left(t u^{+}\right)\left[u^{+}\right]>0$ for $t>0$ small enough (because $\left.u^{+} \not \equiv 0\right)$. Therefore, there exists $\tilde{t} \in] 0,1]$ such that the function $\tilde{u}=-u^{-}+\tilde{t} u^{+}$belongs to $M^{\beta, \varepsilon}$. Moreover, since $f_{\beta, \varepsilon}\left(\tilde{t} u_{n}^{+}\right) \leq f_{\beta, \varepsilon}\left(u_{n}^{+}\right) \forall n \in \mathbb{N}$, we have

$$
\liminf _{n \rightarrow \infty} f_{\beta, \varepsilon}\left(\tilde{t} u_{n}^{+}\right) \leq \liminf _{n \rightarrow \infty} f_{\beta, \varepsilon}\left(u_{n}^{+}\right) .
$$

It follows that

$$
f_{\beta, \varepsilon}(\tilde{u}) \leq \liminf _{n \rightarrow \infty} f_{\beta, \varepsilon}\left(-u_{n}^{-}+\tilde{t} u_{n}^{+}\right) \leq \liminf _{n \rightarrow \infty} f_{\beta, \varepsilon}\left(u_{n}\right)=\inf \left\{f_{\beta, \varepsilon}(u): u \in M^{\beta, \varepsilon}\right\} .
$$

Thus, we can say that the infimum of $f_{\beta, \varepsilon}$ on $M^{\beta, \varepsilon}$ is achieved and that $f_{\beta, \varepsilon}(\tilde{u})=$ $\min _{M^{\beta, \varepsilon}} f_{\beta, \varepsilon}$. 
Let $\beta>\lambda_{1}, \varepsilon>0$ and $u_{\beta, \varepsilon}$ be a minimizing function for $f_{\beta, \varepsilon}$ on $M^{\beta, \varepsilon}$. Our aim is to prove that $u_{\beta, \varepsilon}$ is a constrained critical point for the functional $f_{\beta, \varepsilon}$ constrained on the set $S$, namely that there exists a Lagrange multiplier $\alpha_{\beta, \varepsilon}$ such that

$$
\frac{1}{2} f_{\beta, \varepsilon}^{\prime}\left(u_{\beta, \varepsilon}\right)[\psi]=-\alpha_{\beta, \varepsilon} \int_{\Omega} u_{\beta, \varepsilon}^{-} \psi d x \quad \forall \psi \in H_{0}^{1}(\Omega)
$$

(that is, $u_{\beta, \varepsilon}$ is a weak solution of the equation (2.3)).

Let us point out that, unlike the case of the smooth constraint $\int_{\Omega}\left(u^{-}\right)^{2} d x=1$, for which the Lagrange multipliers theorem applies (and gives the multiplier $\alpha_{\beta, \varepsilon}$ ), the constraint $f_{\beta, \varepsilon}^{\prime}(u)\left[u^{+}\right]=0$ does not satisfy the regularity conditions required in that theorem. However, it is a "natural constraint" in the sense that it does not give rise to Lagrange multipliers.

Notice that (as we observed before) $f_{\beta, \varepsilon}^{\prime}\left(u+t u^{+}\right)\left[u^{+}\right]$is positive for $\left.t \in\right]-1,0[$ and negative for $t>0$. Therefore, $u_{\beta, \varepsilon}$ is the unique maximum point for $f_{\beta, \varepsilon}$ on the set $\left\{u_{\beta, \varepsilon}+t u_{\beta, \varepsilon}^{+}: t \geq-1\right\}$. Then, arguing by contradiction, assume that (2.17) is not satisfied for any choice of the multiplier $\alpha_{\beta, \varepsilon}$ in $\mathbb{R}$. It follows by standard arguments that there exists a continuous map $\eta:]-1,+\infty\left[\rightarrow H_{0}^{1}(\Omega)\right.$ such that $\eta(t)=u_{\beta, \varepsilon}+t u_{\beta, \varepsilon}^{+}$ if $|t|>\frac{1}{2},\left\|\eta(t)^{-}\right\|_{L^{2}(\Omega)}=1, \eta(t)^{+} \not \equiv 0, f_{\beta, \varepsilon}(\eta(t))<f_{\beta, \varepsilon}\left(u_{\beta, \varepsilon}\right) \forall t \geq-1$.

Therefore, we infer that there exists $\bar{t} \in\left[-\frac{1}{2}, \frac{1}{2}\right]$ such that $\eta(\bar{t}) \in M^{\beta, \varepsilon}$, which gives a contradiction because $f_{\beta, \varepsilon}(\eta(\bar{t}))<f_{\beta, \varepsilon}\left(u_{\beta, \varepsilon}\right)$ and $f_{\beta, \varepsilon}\left(u_{\beta, \varepsilon}\right)=\min _{M^{\beta, \varepsilon}} f_{\beta, \varepsilon}$. Thus, we can conclude that there exists a multiplier $\alpha_{\beta, \varepsilon}$ in $\mathbb{R}$ such that (2.17) holds.

Finally, notice that, if in (2.17) we set $\psi=u_{\beta, \varepsilon}^{-}$, we easily obtain $\alpha_{\beta, \varepsilon}=\int_{\Omega}\left|D u_{\beta, \varepsilon}^{-}\right|^{2} d x$, so the proof is complete.

q.e.d.

Now, our aim is to describe the behaviour as $\varepsilon \rightarrow 0$ of the minimizing function $u_{\beta, \varepsilon}$ given by Proposition 2.2.

Proposition 2.3 For all $\beta>\lambda_{1}$ and $\varepsilon>0$, let $u_{\beta, \varepsilon}$ be a minimizing function for the functional $f_{\beta, \varepsilon}$ on the set $M^{\beta, \varepsilon}$ and put $\bar{u}_{\beta, \varepsilon}=-u_{\beta, \varepsilon}^{-}+\left\|u_{\beta, \varepsilon}^{+}\right\|_{L^{2}(\Omega)}^{-1} u_{\beta, \varepsilon}^{+}$. Then, up to a subsequence, $\bar{u}_{\beta, \varepsilon}$ converges in $H_{0}^{1}(\Omega)$, as $\varepsilon \rightarrow 0$, to a function $\bar{u}_{\beta}$ such that $\int_{\Omega}\left|D \bar{u}_{\beta}^{+}\right|^{2} d x=\beta$ and

$$
\begin{aligned}
\int_{\Omega}\left|D \bar{u}_{\beta}^{-}\right|^{2} d x=\min \left\{\int_{\Omega}\left|D u^{-}\right|^{2} d x:\right. & u \in H_{0}^{1}(\Omega), \\
\left\|u^{+}\right\|_{L^{2}(\Omega)} & \left.=\left\|u^{-}\right\|_{L^{2}(\Omega)}=1, \int_{\Omega}\left|D u^{+}\right|^{2} d x=\beta\right\} .
\end{aligned}
$$

Proof Notice that, since $u_{\beta, \varepsilon} \in M^{\beta, \varepsilon}$, we have

$$
\int_{\Omega}\left|D u_{\beta, \varepsilon}^{+}\right|^{2} d x=\int_{\Omega} g_{\beta, \varepsilon}\left(u_{\beta, \varepsilon}\right) u_{\beta, \varepsilon}^{+} d x<\beta \int_{\Omega}\left(u_{\beta, \varepsilon}^{+}\right)^{2} d x \quad \forall \varepsilon>0 .
$$


Therefore, we obtain $\int_{\Omega}\left|D \bar{u}_{\beta, \varepsilon}^{+}\right|^{2} d x<\beta \forall \varepsilon>0$.

Moreover, we have also

$$
\limsup _{\varepsilon \rightarrow 0} \int_{\Omega}\left|D u_{\beta, \varepsilon}^{-}\right|^{2} d x<+\infty .
$$

In fact, since $\beta>\lambda_{1}$, there exists $\hat{u} \in H_{0}^{1}(\Omega)$ such that $\int_{\Omega}\left(\hat{u}^{+}\right)^{2} d x=\int_{\Omega}\left(\hat{u}^{-}\right)^{2} d x=1$, $\int_{\Omega}\left|D \hat{u}^{+}\right|^{2} d x<\beta$. As a consequence, we obtain

$$
f_{\beta, \varepsilon}\left(u_{\beta, \varepsilon}\right) \leq \int_{\Omega}\left|D \hat{u}^{-}\right|^{2} d x+\max \left\{f_{\beta, \varepsilon}\left(t \hat{u}^{+}\right): t \geq 0\right\} \quad \forall \varepsilon>0
$$

and, as $\varepsilon \rightarrow 0$, since $\int_{\Omega}\left|D \hat{u}^{+}\right|^{2} d x<\beta$,

$$
\limsup _{\varepsilon \rightarrow 0} f_{\beta, \varepsilon}\left(u_{\beta, \varepsilon}\right) \leq \int_{\Omega}\left|D \hat{u}^{-}\right|^{2} d x<+\infty
$$

which implies (2.20) because $f_{\beta, \varepsilon}\left(u_{\beta, \varepsilon}\right)=\int_{\Omega}\left|D u_{\beta, \varepsilon}^{-}\right|^{2} d x+f_{\beta, \varepsilon}\left(u_{\beta, \varepsilon}^{+}\right)$with $f_{\beta, \varepsilon}\left(u_{\beta, \varepsilon}^{+}\right)>0$ $\forall \varepsilon>0$.

It follows that, up to a subsequence, $\bar{u}_{\beta, \varepsilon}$ converges as $\varepsilon \rightarrow 0$ to a function $\bar{u}_{\beta} \in H_{0}^{1}(\Omega)$ in $L^{2}(\Omega)$, weakly in $H_{0}^{1}(\Omega)$ and a.e. in $\Omega$.

Let us prove that, indeed, $\bar{u}_{\beta, \varepsilon} \rightarrow \bar{u}_{\beta}$ strongly in $H_{0}^{1}(\Omega)$ as $\varepsilon \rightarrow 0$. In fact, we have

$$
\int_{\Omega}\left|D \bar{u}_{\beta}^{+}\right|^{2} d x=\beta \quad \text { and } \quad \lim _{\varepsilon \rightarrow 0} \int_{\Omega}\left|D u_{\beta, \varepsilon}^{-}\right|^{2} d x=\int_{\Omega}\left|D u_{\beta}^{-}\right|^{2} d x .
$$

For the proof, we argue by contradiction and assume that $\int_{\Omega}\left|D \bar{u}_{\beta}^{+}\right|^{2} d x<\beta$ or (up to a subsequence) $\int_{\Omega}\left|D u_{\beta}^{-}\right|^{2} d x<\lim _{\varepsilon \rightarrow 0} \int_{\Omega}\left|D u_{\beta, \varepsilon}^{-}\right|^{2} d x$. In this case, by slight modifications of the supports of $u_{\beta}^{-}$and $\bar{u}_{\beta}^{+}$, one can construct a function $\tilde{u}_{\beta} \in H_{0}^{1}(\Omega)$ such that $\left\|\tilde{u}_{\beta}^{-}\right\|_{L^{2}(\Omega)}=\left\|\tilde{u}_{\beta}^{+}\right\|_{L^{2}(\Omega)}=1, \int_{\Omega}\left|D \tilde{u}_{\beta}^{+}\right|^{2} d x<\beta$ and $\int_{\Omega}\left|D \tilde{u}_{\beta}^{-}\right|^{2} d x<\lim _{\varepsilon \rightarrow 0} \int_{\Omega}\left|D u_{\beta, \varepsilon}^{-}\right|^{2} d x$. Then, for all $\varepsilon>0$, let us consider the function $\tilde{u}_{\beta, \varepsilon} \in M^{\beta, \varepsilon}$ such that $\tilde{u}_{\beta, \varepsilon}^{-}=\tilde{u}_{\beta}^{-} \forall \varepsilon>0$ and $\tilde{u}_{\beta, \varepsilon}^{+}=t_{\varepsilon} \tilde{u}_{\beta}^{+}$where, for all $\varepsilon>0, t_{\varepsilon}$ is the (unique) positive number such that $f_{\beta, \varepsilon}^{\prime}\left(t_{\varepsilon} \tilde{u}_{\beta}^{+}\right)\left[\tilde{u}_{\beta}^{+}\right]=0$ (such a number $t_{\varepsilon}$ exists because $\int_{\Omega}\left|D \tilde{u}_{\beta}^{+}\right|^{2} d x<\beta$ ).

Thus, we have

$$
f_{\beta, \varepsilon}\left(u_{\beta, \varepsilon}\right)-f_{\beta, \varepsilon}\left(\tilde{u}_{\beta, \varepsilon}\right)=f_{\beta, \varepsilon}\left(u_{\beta, \varepsilon}^{+}\right)-f_{\beta, \varepsilon}\left(\tilde{u}_{\beta, \varepsilon}^{+}\right)+f_{\beta, \varepsilon}\left(u_{\beta, \varepsilon}^{-}\right)-f_{\beta, \varepsilon}\left(\tilde{u}_{\beta, \varepsilon}^{-}\right),
$$

where $f_{\beta, \varepsilon}\left(u_{\beta, \varepsilon}^{+}\right) \geq 0 \forall \varepsilon>0, \lim _{\varepsilon \rightarrow 0} f_{\beta, \varepsilon}\left(\tilde{u}_{\beta, \varepsilon}^{+}\right)=0$ (because $\int_{\Omega}\left|D \tilde{u}_{\beta}^{+}\right|^{2} d x<\beta$ ) and $\lim _{\varepsilon \rightarrow 0} f_{\beta, \varepsilon}\left(u_{\beta, \varepsilon}^{-}\right)>\int_{\Omega}\left|D \tilde{u}_{\beta}^{-}\right|^{2} d x=f_{\beta, \varepsilon}\left(\tilde{u}_{\beta, \varepsilon}^{-}\right) \forall \varepsilon>0$.

It follows that $f_{\beta, \varepsilon}\left(u_{\beta, \varepsilon}\right)>f_{\beta, \varepsilon}\left(\tilde{u}_{\beta, \varepsilon}\right)$ for $\varepsilon>0$ small enough, which gives a contradiction because $\tilde{u}_{\beta, \varepsilon} \in M^{\beta, \varepsilon}$ and $f_{\beta, \varepsilon}\left(u_{\beta, \varepsilon}\right)=\min _{M^{\beta, \varepsilon}} f_{\beta, \varepsilon}$. Thus, we can conclude that $\bar{u}_{\beta, \varepsilon} \rightarrow$ $\bar{u}_{\beta}$ strongly in $H_{0}^{1}(\Omega)$ as $\varepsilon \rightarrow 0$ and that $\int_{\Omega}\left|D \bar{u}_{\beta}^{+}\right|^{2} d x=\beta$.

In a similar way, now we prove (2.18). Arguing again by contradiction, assume that there exists a function $v \in H_{0}^{1}(\Omega)$ such that $\left\|v^{+}\right\|_{L^{2}(\Omega)}=\left\|v^{-}\right\|_{L^{2}(\Omega)}=1, \int_{\Omega}\left|D v^{+}\right|^{2} d x=$ $\beta$ and $\int_{\Omega}\left|D v^{-}\right|^{2} d x<\int_{\Omega}\left|D \bar{u}_{\beta}^{-}\right|^{2} d x$. 
In this case, by slight modifications of the supports of $v^{+}$and $v^{-}$, one can find $\hat{v}_{\beta} \in$ $H_{0}^{1}(\Omega)$ such that $\left\|\hat{v}_{\beta}^{+}\right\|_{L^{2}(\Omega)}=\left\|\hat{v}_{\beta}^{-}\right\|_{L^{2}(\Omega)}=1, \int_{\Omega}\left|D \hat{v}_{\beta}^{+}\right|^{2} d x<\beta$ and $\int_{\Omega}\left|D \hat{v}_{\beta}^{-}\right|^{2} d x<$ $\int_{\Omega}\left|D \bar{u}_{\beta}^{-}\right|^{2} d x$.

Since $\int_{\Omega}\left|D \hat{v}_{\beta}^{+}\right|^{2} d x<\beta$ and $\left\|\hat{v}_{\beta}^{+}\right\|_{L^{2}(\Omega)}=1$, it follows that for all $\varepsilon>0$ there exists $\hat{t}_{\varepsilon}>0$ such that $f_{\beta, \varepsilon}^{\prime}\left(\hat{t}_{\varepsilon} \hat{v}_{\beta}^{+}\right)\left[\hat{v}_{\beta}^{+}\right]=0$, namely, the function $\hat{v}_{\beta, \varepsilon}=-\hat{v}_{\beta}^{-}+\hat{t}_{\varepsilon} \hat{v}_{\beta}^{+}$belongs to $M^{\beta, \varepsilon}$.

Then, by direct computation, we obtain

$$
f_{\beta, \varepsilon}\left(u_{\beta, \varepsilon}\right)-f_{\beta, \varepsilon}\left(\hat{v}_{\beta, \varepsilon}\right)=f_{\beta, \varepsilon}\left(u_{\beta, \varepsilon}^{-}\right)-f_{\beta, \varepsilon}\left(\hat{v}_{\beta, \varepsilon}^{-}\right)+f_{\beta, \varepsilon}\left(u_{\beta, \varepsilon}^{+}\right)-f_{\beta, \varepsilon}\left(\hat{v}_{\beta, \varepsilon}^{+}\right) \text {, }
$$

where $f_{\beta, \varepsilon}\left(u_{\beta, \varepsilon}^{+}\right) \geq 0, f_{\beta, \varepsilon}\left(\hat{v}_{\beta, \varepsilon}^{-}\right)=\int_{\Omega}\left|D \hat{v}_{\beta}^{-}\right|^{2} d x \forall \varepsilon>0, \lim _{\varepsilon \rightarrow 0} f_{\beta, \varepsilon}\left(\hat{v}_{\beta, \varepsilon}^{+}\right)=0$ and

$$
\lim _{\varepsilon \rightarrow 0} f_{\beta, \varepsilon}\left(u_{\beta, \varepsilon}^{-}\right)=\int_{\Omega}\left|D \bar{u}_{\beta}^{-}\right|^{2} d x>\int_{\Omega}\left|D \hat{v}_{\beta}^{-}\right|^{2} d x .
$$

It follows that $f_{\beta, \varepsilon}\left(u_{\beta, \varepsilon}\right)>f_{\beta, \varepsilon}\left(\hat{v}_{\beta, \varepsilon}\right)$ for $\varepsilon>0$ small enough; so we have again a contradiction because $\hat{v}_{\beta, \varepsilon} \in M^{\beta, \varepsilon}$ and $f_{\beta, \varepsilon}\left(u_{\beta, \varepsilon}\right)=\min _{M^{\beta, \varepsilon}} f_{\beta, \varepsilon} \forall \varepsilon>0$.

q.e.d.

Proposition 2.4 For all $\beta>\lambda_{1}$ and $\varepsilon>0$, let $u_{\beta, \varepsilon}$ be a minimizing function for the functional $f_{\beta, \varepsilon}$ on the set $M^{\beta, \varepsilon}$. Then, as $\varepsilon \rightarrow 0$ (up to a subsequence) $u_{\beta, \varepsilon}$ converges in $H_{0}^{1}(\Omega)$ to a sign changing function $u_{\beta}$ which solves the equation

$$
\Delta u_{\beta}-\alpha_{\beta} u_{\beta}^{-}+\beta u_{\beta}^{+}=0 \quad \text { in } \Omega,
$$

where $\alpha_{\beta}$ is the positive number introduced in Theorem 2.1. Moreover, the function $\bar{u}_{\beta}=-u_{\beta}^{-}+\left\|u_{\beta}^{+}\right\|_{L^{2}(\Omega)}^{-1} u_{\beta}^{+}$is a minimizing function for $\alpha_{\beta}$ (see (2.1)) and $\lambda_{1}<\alpha_{\beta} \leq \alpha$ for every $\alpha>\lambda_{1}$ such that $(\alpha, \beta) \in \Sigma$.

Proof As we proved in Proposition 2.2, for all $\beta>\lambda_{1}$ and $\varepsilon>0, u_{\beta, \varepsilon}$ is a weak solution of the equation

$$
\Delta u_{\beta, \varepsilon}-\alpha_{\beta, \varepsilon} u_{\beta, \varepsilon}^{-}+g_{\beta, \varepsilon}\left(u_{\beta, \varepsilon}\right)=0 \quad \text { in } \Omega
$$

where $\alpha_{\beta, \varepsilon}=\int_{\Omega}\left|D u_{\beta, \varepsilon}^{-}\right|^{2} d x$.

Moreover, by Proposition 2.3, $\bar{u}_{\beta, \varepsilon}=-u_{\beta, \varepsilon}^{-}+\left\|u_{\beta, \varepsilon}^{+}\right\|_{L^{2}(\Omega)}^{-1} u_{\beta, \varepsilon}^{+}$converges, as $\varepsilon \rightarrow 0$, to a function $\bar{u}_{\beta}$ in $H_{0}^{1}(\Omega)$.

Let us prove that

$$
\liminf _{\varepsilon \rightarrow 0}\left\|u_{\beta, \varepsilon}^{+}\right\|_{L^{2}(\Omega)}>0 \quad \forall \beta>\lambda_{1} .
$$


Arguing by contradiction, assume that (up to a subsequence) $\lim _{\varepsilon \rightarrow 0}\left\|u_{\beta, \varepsilon}^{+}\right\|_{L^{2}(\Omega)}=0$ for some $\beta>\lambda_{1}$. In this case, since $f_{\beta, \varepsilon}^{\prime}\left(u_{\beta, \varepsilon}\right)\left[u_{\beta, \varepsilon}^{+}\right]=0$, we infer that $u_{\beta, \varepsilon}^{+} \rightarrow 0$ in $H_{0}^{1}(\Omega)$ as $\varepsilon \rightarrow 0$. Therefore, if we let $\varepsilon \rightarrow 0$, from (2.28) we obtain

$$
\int_{\Omega}\left[D \bar{u}_{\beta}^{-} D \psi-\bar{\alpha}_{\beta} \bar{u}_{\beta}^{-} \psi\right] d x=0 \quad \forall \psi \in H_{0}^{1}(\Omega),
$$

where $\bar{\alpha}_{\beta}=\int_{\Omega}\left|D \bar{u}_{\beta}^{-}\right|^{2} d x$. Thus, we have a contradiction because $D \bar{u}_{\beta}^{-} \not \equiv 0$ on $\Omega \cap$ $\partial\left(\operatorname{supp} \bar{u}_{\beta}^{-}\right)$.

Now, let us prove that

$$
\limsup _{\varepsilon \rightarrow 0}\left\|u_{\beta, \varepsilon}^{+}\right\|_{L^{2}(\Omega)}<+\infty \quad \forall \beta>\lambda_{1} .
$$

Arguing again by contradiction, assume that (up to a subsequence) $\lim _{\varepsilon \rightarrow 0}\left\|u_{\beta, \varepsilon}^{+}\right\|_{L^{2}(\Omega)}=$ $+\infty$ for some $\beta>\lambda_{1}$. Then, as $\varepsilon \rightarrow 0$, from (2.28) we obtain

$$
\int_{\Omega}\left[D \bar{u}_{\beta}^{+} D \psi-\beta \bar{u}_{\beta}^{+} \psi\right] d x=0 \quad \forall \psi \in H_{0}^{1}(\Omega) .
$$

Thus, we again have a contradiction because $D \bar{u}_{\beta}^{+} \not \equiv 0$ on $\Omega \cap \partial\left(\operatorname{supp} \bar{u}_{\beta}^{+}\right)$.

Therefore, we can say that for all $\beta>\lambda_{1}$ (up to a subsequence) $u_{\beta, \varepsilon}$ converges as $\varepsilon \rightarrow 0$ to a sign changing function $u_{\beta}$ strongly in $H_{0}^{1}(\Omega)$. Moreover, if we let $\varepsilon \rightarrow 0$ in (2.28), because of the minimality property (2.18) given by Proposition 2.3 , we infer that $u_{\beta}$ is a weak solution of the Dirichlet problem

$$
\Delta u_{\beta}-\alpha_{\beta} u_{\beta}^{-}+\beta u_{\beta}^{+}=0 \quad \text { in } \Omega, \quad u_{\beta}=0 \quad \text { on } \partial \Omega .
$$

It follows that $\int_{\Omega}\left|D u_{\beta}^{+}\right|^{2} d x=\beta \int_{\Omega}\left(u_{\beta}^{+}\right)^{2} d x>0$ and $\int_{\Omega}\left|D u_{\beta}^{-}\right|^{2} d x=\alpha_{\beta} \forall \beta>\lambda_{1}$ (notice that $\int_{\Omega}\left|D u_{\beta}^{-}\right|^{2} d x>\lambda_{1}$ because $\left.u_{\beta}^{+} \not \equiv 0\right)$. So we can say that the function $\bar{u}_{\beta}=$ $-u_{\beta}^{-}+\left\|u_{\beta}^{+}\right\|_{L^{2}(\Omega)}^{-1} u_{\beta}^{+}$is a minimizing function for the infimum in (2.1) and $\alpha_{\beta} \leq \alpha$ for every $\alpha>\lambda_{1}$ such that $(\alpha, \beta) \in \Sigma$, which completes the proof.

q.e.d.

Proposition 2.5 For all $\beta>\lambda_{1}$, let $\alpha_{\beta}$ be the positive number introduced in Theorem 2.1. Then $\alpha_{\beta}$ is continuous and strictly decreasing with respect to $\beta$ in $] \lambda_{1},+\infty[$. Moreover, $\lim _{\beta \rightarrow+\infty} \alpha_{\beta}=\lambda_{1}$ and $\lim _{\beta \rightarrow \lambda_{1}} \alpha_{\beta}=+\infty$.

Proof First, let us prove that $\alpha_{\beta}$ depends continuously on $\beta$ in $] \lambda_{1},+\infty[$, namely, $\left.\lim _{\beta \rightarrow \bar{\beta}} \alpha_{\beta}=\alpha_{\bar{\beta}} \forall \bar{\beta} \in\right] \lambda_{1},+\infty[$.

Let us set $\bar{u}_{\beta}=-u_{\beta}^{-}+\left\|u_{\beta}^{+}\right\|_{L^{2}(\Omega)}^{-1} u_{\beta}^{+} \forall \beta>\lambda_{1}$, where $u_{\beta}$ is the eigenfunction, corresponding to the pair $\left(\alpha_{\beta}, \beta\right)$, given by Proposition 2.4 . 
In order to prove that $\liminf _{\beta \rightarrow \bar{\beta}} \alpha_{\beta} \geq \alpha_{\bar{\beta}}$, we argue by contradiction and assume that there exists a sequence $\left(\beta_{n}\right)_{n}$ such that $\lim _{n \rightarrow \infty} \beta_{n}=\bar{\beta}$ and $\lim _{n \rightarrow \infty} \alpha_{\beta_{n}}<\alpha_{\bar{\beta}}$.

Since $\alpha_{\beta_{n}}=\int_{\Omega}\left|D \bar{u}_{\beta_{n}}^{-}\right|^{2} d x$ and $\int_{\Omega}\left|D \bar{u}_{\beta_{n}}^{+}\right|^{2} d x=\beta_{n} \forall n \in \mathbb{N}$, it follows that the sequence $\left(\bar{u}_{\beta_{n}}\right)_{n}$ is bounded in $H_{0}^{1}(\Omega)$, so (up to a subsequence) it converges to a function $\hat{u}$ in $L^{2}(\Omega)$, weakly in $H_{0}^{1}(\Omega)$ and a.e. in $\Omega$. Then, we have

$$
\lim _{n \rightarrow \infty} \int_{\Omega}\left|D \bar{u}_{\beta_{n}}^{-}\right|^{2} d x \geq \int_{\Omega}\left|D \hat{u}^{-}\right|^{2} d x \quad \text { and } \quad \int_{\Omega}\left|D \hat{u}^{+}\right|^{2} d x \leq \lim _{n \rightarrow \infty} \int_{\Omega}\left|D \bar{u}_{\beta_{n}}^{+}\right|^{2} d x=\bar{\beta} .
$$

As a consequence, there exists a function $\hat{v} \in H_{0}^{1}(\Omega)$ such that $0 \leq \hat{v} \leq \hat{u}^{+}$in $\Omega$ and $\int_{\Omega}|D \hat{v}|^{2} d x=\bar{\beta} \int_{\Omega} \hat{v}^{2} d x>0$.

Therefore, by (2.1) we have

$$
\alpha_{\bar{\beta}} \leq \int_{\Omega}\left|D \hat{u}^{-}\right|^{2} d x \leq \lim _{n \rightarrow \infty} \int_{\Omega}\left|D \bar{u}_{\beta_{n}}^{-}\right|^{2} d x=\lim _{n \rightarrow \infty} \alpha_{\beta_{n}},
$$

which is a contradiction. Thus we can say that $\liminf _{\beta \rightarrow \bar{\beta}} \alpha_{\beta} \geq \alpha_{\bar{\beta}}$.

Now, let us prove that $\lim _{\sup _{\beta \rightarrow \bar{\beta}}} \alpha_{\beta} \leq \alpha_{\bar{\beta}}$. By slight perturbations of the function $\bar{u}_{\bar{\beta}}$, one can construct $\forall \beta>\lambda_{1}$ a function $\tilde{u}_{\beta} \in H_{0}^{1}(\Omega)$ such that $\left\|\tilde{u}_{\beta}^{+}\right\|_{L^{2}(\Omega)}=\left\|\tilde{u}_{\beta}^{-}\right\|_{L^{2}(\Omega)}=$ $1, \int_{\Omega}\left|D \tilde{u}_{\beta}^{+}\right|^{2} d x=\beta \forall \beta>\lambda_{1}$ and $\tilde{u}_{\beta}^{-} \rightarrow \bar{u}_{\bar{\beta}}^{-}$in $H_{0}^{1}(\Omega)$ as $\beta \rightarrow \bar{\beta}$. It follows that $\alpha_{\beta} \leq \int_{\Omega}\left|D \tilde{u}_{\beta}^{-}\right|^{2} d x \forall \beta>\lambda_{1}$ and

$$
\limsup _{\beta \rightarrow \bar{\beta}} \alpha_{\beta} \leq \lim _{\beta \rightarrow \bar{\beta}} \int_{\Omega}\left|D \tilde{u}_{\beta}^{-}\right|^{2} d x=\int_{\Omega}\left|D \bar{u}_{\bar{\beta}}^{-}\right|^{2} d x=\alpha_{\bar{\beta}}
$$

Thus, we can conclude that $\alpha_{\beta}$ depends continuously on $\beta$ in $] \lambda_{1},+\infty[$.

Now, we prove that $\alpha_{\beta}$ is strictly decreasing with respect to $\beta$ in $] \lambda_{1},+\infty[$. Let us consider $\beta_{1}$ and $\beta_{2}$ in $] \lambda_{1},+\infty\left[\right.$ such that $\beta_{1}<\beta_{2}$. Since $\alpha_{\beta_{1}}=\int_{\Omega}\left|D u_{\beta_{1}}^{-}\right|^{2} d x$ and $\beta_{1}<\beta_{2}$, there exists $\check{u}_{\beta_{2}} \in H_{0}^{1}(\Omega)\left(\right.$ with $\operatorname{supp}\left(\check{u}_{\beta_{2}}^{+}\right) \subseteq \operatorname{supp}\left(u_{\beta_{1}}^{+}\right)$and $\operatorname{supp}\left(\check{u}_{\beta_{2}}^{-}\right) \supseteq$ $\left.\operatorname{supp}\left(u_{\beta_{1}}^{-}\right)\right)$such that $\left\|\check{u}_{\beta_{2}}^{+}\right\|_{L^{2}(\Omega)}=\left\|\check{u}_{\beta_{2}}^{-}\right\|_{L^{2}(\Omega)}=1, \int_{\Omega}\left|D \check{u}_{\beta_{2}}^{+}\right|^{2} d x=\beta_{2}, \int_{\Omega}\left|D \check{u}_{\beta_{2}}^{-}\right|^{2} d x<$ $\int_{\Omega}\left|D u_{\beta_{1}}^{-}\right|^{2} d x=\alpha_{\beta_{1}}$.

Therefore, taking into account Proposition 2.3 (see (2.18)), we obtain

$$
\begin{gathered}
\alpha_{\beta_{2}}=\min \left\{\int_{\Omega}\left|D u^{-}\right|^{2} d x: u \in H_{0}^{1}(\Omega),\left\|u^{+}\right\|_{L^{2}(\Omega)}=\left\|u^{-}\right\|_{L^{2}(\Omega)}=1,\right. \\
\left.\int_{\Omega}\left|D u^{+}\right|^{2} d x=\beta_{2}\right\}<\int_{\Omega}\left|D u_{\beta_{1}}^{-}\right|^{2} d x
\end{gathered}
$$

that is $\alpha_{\beta_{2}}<\alpha_{\beta_{1}}$.

Let us prove that $\lim _{\beta \rightarrow+\infty} \alpha_{\beta}=\lambda_{1}$. Since $\alpha_{\beta}$ is decreasing with respect to $\beta$, from Proposition 2.3 we obtain

$$
\lambda_{1}<\alpha_{\beta}=\inf \left\{\int_{\Omega}\left|D u^{-}\right|^{2} d x: u \in H_{0}^{1}(\Omega),\left\|u^{+}\right\|_{L^{2}(\Omega)}=\left\|u^{-}\right\|_{L^{2}(\Omega)}=1,\right.
$$




$$
\left.\int_{\Omega}\left|D u^{+}\right|^{2} d x \leq \beta\right\}
$$

As $\beta \rightarrow+\infty$, it follows that

$$
\begin{aligned}
\lambda_{1} & \leq \lim _{\beta \rightarrow+\infty} \alpha_{\beta}=\inf \left\{\alpha_{\beta}: \beta>\lambda_{1}\right\} \\
& \leq \inf \left\{\int_{\Omega}\left|D u^{-}\right|^{2} d x: u \in H_{0}^{1}(\Omega),\left\|u^{+}\right\|_{L^{2}(\Omega)}=\left\|u^{-}\right\|_{L^{2}(\Omega)}=1\right\} \\
& \left.\leq \int_{\Omega_{r}}\left|D e_{1}\left(\Omega_{r}\right)\right|^{2} d x \quad \forall r \in\right] 0, \frac{1}{2} \operatorname{diam}(\Omega)[,
\end{aligned}
$$

where $\Omega_{r}$ and $e_{1}\left(\Omega_{r}\right)$ are defined in the following way: we fix $x_{0} \in \partial \Omega$, set $\Omega_{r}=$ $\Omega \backslash \bar{B}\left(x_{0}, r\right)$ and denote by $e_{1}\left(\Omega_{r}\right)$ the positive eigenfunction, normalized in $L^{2}\left(\Omega_{r}\right)$, corresponding to the first eigenvalue of the operator $-\Delta$ in $H_{0}^{1}\left(\Omega_{r}\right)$. Then, as $r \rightarrow 0$, we obtain $\lim _{\beta \rightarrow+\infty} \alpha_{\beta}=\lambda_{1}$. Notice that, as a consequence, we have also $\lim _{\beta \rightarrow \lambda_{1}} \alpha_{\beta}=$ $+\infty$, because the set $\left\{\left(\alpha_{\beta}, \beta\right) \in \mathbb{R}^{2}: \beta>\lambda_{1}\right\}$ (the first nontrivial curve of $\Sigma$ ) is symmetric with respect to the line $\left\{(\alpha, \beta) \in \mathbb{R}^{2}: \alpha=\beta\right\}$ (since a pair $(\alpha, \beta) \in \Sigma$ if and only if $(\beta, \alpha) \in \Sigma)$.

q.e.d.

Proof of Theorem 2.1 For all $\beta>\lambda_{1}$ and $\varepsilon>0$, let $u_{\beta, \varepsilon}$ be a minimizing function for the functional $f_{\beta, \varepsilon}$ on the set $M^{\beta, \varepsilon}$ (here we use Proposition 2.2). From Propositions 2.3 and 2.4 we deduce that, as $\varepsilon \rightarrow 0, u_{\beta, \varepsilon}$ converges in $H_{0}^{1}(\Omega)$ to a function $u_{\beta}$ such that $u_{\beta}^{+} \not \equiv 0$ and $\left\|u_{\beta}^{-}\right\|_{L^{2}(\Omega)}=1$, satisfying the equation $\Delta u_{\beta}-\alpha_{\beta} u_{\beta}^{-}+\beta u_{\beta}^{+}=0$ in $\Omega$, with $\alpha_{\beta}=\int_{\Omega}\left|D u_{\beta}^{-}\right|^{2} d x>\lambda_{1}$. Thus $\left(\alpha_{\beta}, \beta\right) \in \Sigma$. Moreover, from Proposition 2.3 we infer that the function $\bar{u}_{\beta}=-u_{\beta}^{-}+\left\|u_{\beta}^{+}\right\|_{L^{2}(\Omega)}^{-1} u_{\beta}^{+}$is a minimizing function for (2.1) and $\alpha_{\beta} \leq \alpha$ for every $\alpha>\lambda_{1}$ such that $(\alpha, \beta) \in \Sigma$. Notice that the eigenfunction $u_{\beta}$ corresponding to the pair $\left(\alpha_{\beta}, \beta\right)$ may be written as $u_{\beta}=-\bar{u}_{\beta}^{-}+\mu_{\beta} \bar{u}_{\beta}^{+}$with $\mu_{\beta}=\left\|u_{\beta}^{+}\right\|_{L^{2}(\Omega)}$.

By Proposition 2.5, we know that $\alpha_{\beta}$ is continuous and strictly decreasing with respect to $\beta$ in $] \lambda_{1},+\infty\left[\right.$ and that $\lim _{\beta \rightarrow+\infty} \alpha_{\beta}=\lambda_{1}, \lim _{\beta \rightarrow \lambda_{1}} \alpha_{\beta}=+\infty$. As a consequence, we can infer that $\alpha_{\lambda_{2}}=\lambda_{2}$. In fact, because of the minimality property (2.1), since $\left(\lambda_{2}, \lambda_{2}\right) \in \Sigma$, we have $\alpha_{\lambda_{2}} \leq \lambda_{2}$. Arguing by contradiction, assume that $\alpha_{\lambda_{2}}<\lambda_{2}$. Then, the continuous curve $\left\{\left(\alpha_{\beta}, \beta\right) \in \mathbb{R}^{2}: \beta \in\right] \lambda_{1},+\infty[\}$ meets the line $\left\{(\alpha, \beta) \in \mathbb{R}^{2}\right.$ : $\alpha=\beta\}$ in a point $(\bar{\lambda}, \bar{\lambda}) \in \mathbb{R}^{2}$ with $\lambda_{1}<\bar{\lambda}<\lambda_{2}$, which is impossible because $\bar{\lambda}$ must be an eigenvalue for the Laplace operator $-\Delta$ in $H_{0}^{1}(\Omega)$. Thus, we can conclude that $\alpha_{\lambda_{2}}=\lambda_{2}$.

Now let us prove that, as $\beta \rightarrow+\infty, u_{\beta}^{-} \rightarrow e_{1}$ in $H_{0}^{1}(\Omega)$. In fact, $\left\|u_{\beta}^{-}\right\|_{L^{2}(\Omega)}=1$ and $\alpha_{\beta}=\int_{\Omega}\left|D u_{\beta}^{-}\right|^{2} d x \forall \beta>\lambda_{1}$. Since $\lim _{\beta \rightarrow+\infty} \alpha_{\beta}=\lambda_{1}, u_{\beta}^{-}$converges to a function $\bar{u} \in H_{0}^{1}(\Omega)$ in $L^{2}(\Omega)$, weakly in $H_{0}^{1}(\Omega)$ and a.e. in $\Omega$; moreover,

$$
\lambda_{1}=\lim _{\beta \rightarrow+\infty} \int_{\Omega}\left|D u_{\beta}^{-}\right|^{2} d x \leq \int_{\Omega}|D \bar{u}|^{2} d x
$$


because $\bar{u} \in H_{0}^{1}(\Omega)$ and $\|\bar{u}\|_{L^{2}(\Omega)}=1$. It follows that $u_{\beta}^{-} \rightarrow \bar{u}$ strongly in $H_{0}^{1}(\Omega)$ and $\bar{u}=e_{1}$. Finally, taking into account that a function $u$ satisfies (1.3) if and only if $-u$ satisfies (1.3) with $(\beta, \alpha)$ in place of $(\alpha, \beta)$, we infer that $\left\|u_{\beta}^{+}\right\|_{L^{2}(\Omega)}^{-1} u_{\beta}^{+} \rightarrow e_{1}$ in $H_{0}^{1}(\Omega)$, as $\beta \rightarrow \lambda_{1}$, so the proof is complete.

q.e.d.

\section{Comparison with other curves of $\Sigma$ and final re- marks}

Now, our aim is to use the variational characterization of the first nontrivial curve of the Fučík spectrum $\Sigma$, obtained in Section 2, in order to prove that this curve is distinct from all the infinitely many curves of $\Sigma$ obtained in previous papers (see [36, 37] and the references therein).

In the next theorem we gather the main results presented in [36] and [37].

Theorem 3.1 Let $\Omega$ be a bounded connected domain of $\mathbb{R}^{N}$ with $N \geq 2$. Then, there exists a nondecreasing sequence $\left(b_{k}\right)_{k}$ of positive numbers, having the following properties. For every positive integer $k$ and for all $\beta>b_{k}$, there exist $\alpha_{k, \beta}>\lambda_{1}$ and $u_{k, \beta} \in$ $H_{0}^{1}(\Omega)$, with $u_{k, \beta}^{+} \not \equiv 0$ and $u_{k, \beta}^{-} \not \equiv 0$, such that the equation $\Delta u_{k, \beta}-\alpha_{k, \beta} u_{k, \beta}^{-}+\beta u_{k, \beta}^{+}=0$ in $\Omega$ is satisfied for all $\beta>b_{k}$. Moreover, for every positive integer $k, \alpha_{k, \beta}$ depends continuously on $\beta$ in $] b_{k},+\infty\left[, \alpha_{k, \beta}<\alpha_{k+1, \beta} \forall \beta>b_{k+1}, \alpha_{k, \beta} \rightarrow \lambda_{1}\right.$ as $\beta \rightarrow+\infty$, while $u_{k, \beta} \rightarrow-e_{1}$ in $H_{0}^{1}(\Omega)$.

Thus, the continuous curves $\left\{\left(\alpha_{k, \beta}, \beta\right) \in \mathbb{R}^{2}: \beta>b_{k}\right\}$ and $\left\{\left(\beta, \alpha_{k, \beta}\right) \in \mathbb{R}^{2}: \beta>b_{k}\right\}$ are included in the nontrivial part of the Fučík spectrum $\Sigma$ for all $k \in \mathbb{N}$ and, as the first nontrivial curve of $\Sigma$, are all asymptotic to the lines $\left\{\lambda_{1}\right\} \times \mathbb{R}$ and $\mathbb{R} \times\left\{\lambda_{1}\right\}$.

In addition, these curves and the corresponding eigenfunctions have the properties described in the following proposition (see $[36,37]$ and the references therein).

Proposition 3.2 Let $\Omega$ be a bounded connected domain of $\mathbb{R}^{N}$ with $N \geq 2$. For every positive integer $k$, let $b_{k}>0$ and, for $\beta>b_{k}$, let $\alpha_{k, \beta}>\lambda_{1}$ and $u_{k, \beta} \in H_{0}^{1}(\Omega)$ be the positive number and the function given by Theorem 3.1. Then, the following properties hold. There exist $r>0$ and, for all $k \geq 1$ and $\beta>b_{k}, k$ points $x_{1, \beta}, \ldots, x_{k, \beta}$ in $\Omega$ such that the balls $B\left(x_{1, \beta}, \frac{r}{\sqrt{\beta}}\right), \ldots, B\left(x_{k, \beta}, \frac{r}{\sqrt{\beta}}\right)$ are pairwise disjoint and all included in $\Omega, u_{k, \beta}(x) \leq 0 \forall x \in \Omega \backslash \cup_{i=1}^{k} B\left(x_{i, \beta}, \frac{r}{\sqrt{\beta}}\right)$ and $u_{k, \beta}^{+} \not \equiv 0$ in $B\left(x_{i, \beta}, \frac{r}{\sqrt{\beta}}\right) \forall i \in\{1, \ldots, k\}$. As $\beta \rightarrow+\infty$, we have

$$
\lim _{\beta \rightarrow+\infty} e_{1}\left(x_{i, \beta}\right)=\max _{\Omega} e_{1} \quad \forall i \in\{1, \ldots, k\}
$$


and

$$
\lim _{\beta \rightarrow+\infty} \sqrt{\beta}\left|x_{i, \beta}-x_{j, \beta}\right|=\infty \quad \text { for } i \neq j .
$$

If $\forall k \in \mathbb{N}, \forall i \in\{1, \ldots, k\}, \forall \beta>b_{k}$, we set $s_{i, k, \beta}=\sup \left\{u_{k, \beta}(x): x \in B\left(x_{i, \beta}, \frac{r}{\sqrt{\beta}}\right)\right\}$ and define $U_{i, k, \beta}(x)=s_{i, k, \beta}^{-1} u_{k, \beta}\left(\frac{x}{\sqrt{\beta}}+x_{i, \beta}\right) \forall x \in \sqrt{\beta}\left(\Omega-x_{i, \beta}\right)$, then the rescaled function $U_{i, k, \beta}$ converges as $\beta \rightarrow+\infty$ to the radial solution $U$ of the problem

$$
\Delta U+U^{+}=0 \quad \text { in } \mathbb{R}^{N}, \quad U(0)=1
$$

and the convergence is uniform on the compact subsets of $\mathbb{R}^{N}$.

If $N \geq 3$, we have

$$
\lim _{\beta \rightarrow+\infty} \beta^{\frac{N-2}{2}}\left(\alpha_{k, \beta}-\lambda_{1}\right)=\operatorname{cap}\left(\bar{r}_{1}\right)\left(\max _{\Omega} e_{1}\right)^{2} k
$$

where $\bar{r}_{1}$ is the radius of the balls in $\mathbb{R}^{N}$ for which the first eigenvalue of $-\Delta$ in $H_{0}^{1}$ is equal to 1 and $\operatorname{cap}\left(\bar{r}_{1}\right)$ denotes the capacity of these balls.

Finally, in the case $N=2$ we have

$$
\lim _{\beta \rightarrow+\infty} \lg \beta\left(\alpha_{k, \beta}-\lambda_{1}\right)=4 \pi\left(\max _{\Omega} e_{1}\right)^{2} k
$$

In Proposition 2.5 we proved that $\alpha_{\beta}$ converges to $\lambda_{1}$ as $\beta \rightarrow+\infty$; now, we need to estimate the rate of convergence.

Proposition 3.3 For all $\beta>\lambda_{1}$, let $\alpha_{\beta}$ be the positive number introduced in Theorem 2.1. Then, for $N \geq 3$ we have

$$
\lim _{\beta \rightarrow+\infty} \beta^{\frac{N-2}{2}}\left(\alpha_{\beta}-\lambda_{1}\right)=0
$$

while, for $N=2$,

$$
\lim _{\beta \rightarrow+\infty} \lg \beta\left(\alpha_{\beta}-\lambda_{1}\right)=0
$$

Proof Let $\bar{r}_{1}$ be the positive number introduced in Proposition 3.2. For all $y \in$ $\Omega$, let us consider the function $\bar{u}_{\beta, y} \in H_{0}^{1}(\Omega)$, defined as follows. First notice that $B\left(y, \frac{\bar{r}_{1}}{\sqrt{\beta}}\right) \subseteq \Omega$ for $\beta$ large enough and the first eigenvalue of $-\Delta$ in $H_{0}^{1}\left(B\left(y, \frac{\bar{r}_{1}}{\sqrt{\beta}}\right)\right)$ is equal to $\beta$ (because of the choice of $\bar{r}_{1}$ ). Then, for $\beta>0$ large enough, in the ball $B\left(y, \frac{\bar{r}_{1}}{\sqrt{\beta}}\right)$ we define $\bar{u}_{\beta, y}$ to be the positive eigenfunction corresponding to the first eigenvalue of $-\Delta$ in $H_{0}^{1}\left(B\left(y, \frac{\bar{r}_{1}}{\sqrt{\beta}}\right)\right)$, normalized in $L^{2}\left(B\left(y, \frac{\bar{r}_{1}}{\sqrt{\beta}}\right)\right)$. Now, in order to define $\bar{u}_{\beta, y}$ in $\Omega \backslash B\left(y, \frac{\bar{r}_{1}}{\sqrt{\beta}}\right)$, set $\varepsilon_{\beta}=\beta^{-q}$ with $\left.q \in\right] \frac{1}{2}-\frac{1}{N}, \frac{1}{2}\left[\right.$ if $N \geq 3$ and $\varepsilon_{\beta}=\frac{1}{\lg \beta}$ if 
$N=2$. Then, for $\beta>0$ large enough, consider the function $\tilde{u}_{\beta, y}$ in $H_{0}^{1}\left(\Omega \backslash \bar{B}\left(y, \frac{\bar{r}_{1}}{\sqrt{\beta}}\right)\right)$, such that $\Delta \tilde{u}_{\beta, y}=0$ in the annulus $A\left(y, \frac{\bar{r}_{1}}{\sqrt{\beta}}, \frac{\bar{r}_{1}}{\sqrt{\beta}}+\varepsilon_{\beta}\right)=B\left(y, \frac{\bar{r}_{1}}{\sqrt{\beta}}+\varepsilon_{\beta}\right) \backslash \bar{B}\left(y, \frac{\bar{r}_{1}}{\sqrt{\beta}}\right)$ and $\tilde{u}_{\beta, y}(x)=-e_{1}(x) \forall x \in \Omega \backslash B\left(y, \frac{\bar{r}_{1}}{\sqrt{\beta}}+\varepsilon_{\beta}\right)$. Finally, we complete the definition of $\bar{u}_{\beta, y}$ by setting $\bar{u}_{\beta, y}(x)=\left\|\tilde{u}_{\beta, y}\right\|_{L^{2}\left(\Omega \backslash \bar{B}\left(y, \bar{r}_{1} / \sqrt{\beta}\right)\right)}^{-1} \tilde{u}_{\beta, y}(x) \forall x \in \Omega \backslash \bar{B}\left(y, \frac{\bar{r}_{1}}{\sqrt{\beta}}\right)$.

Since $\left\|\bar{u}_{\beta, y}^{+}\right\|_{L^{2}(\Omega)}=\left\|\bar{u}_{\beta, y}^{-}\right\|_{L^{2}(\Omega)}=1$ and $\int_{\Omega}\left|D \bar{u}_{\beta, y}^{+}\right|^{2} d x=\beta$, taking into account $(2.1)$ we infer that $\alpha_{\beta} \leq \int_{\Omega}\left|D \bar{u}_{\beta, y}^{-}\right|^{2} d x$ for $\beta>0$ large enough so that $B\left(y, \frac{\bar{r}_{1}}{\sqrt{\beta}}+\varepsilon_{\beta}\right) \subseteq \Omega$. Let us estimate the integral $\int_{\Omega}\left|D \bar{u}_{\beta, y}^{-}\right|^{2} d x$. We have

$$
\int_{\Omega}\left|D \bar{u}_{\beta, y}^{-}\right|^{2} d x=\left\|\tilde{u}_{\beta, y}\right\|_{L^{2}\left(\Omega \backslash \bar{B}\left(y, \bar{r}_{1} / \sqrt{\beta}\right)\right)}^{-2} \int_{\Omega \backslash \bar{B}\left(y, \frac{\bar{r}_{1}}{\sqrt{\beta}}\right)}\left|D \tilde{u}_{\beta, y}\right|^{2} d x
$$

where

$$
\left\|\tilde{u}_{\beta, y}\right\|_{L^{2}\left(\Omega \backslash \bar{B}\left(y, \bar{r}_{1} / \sqrt{\beta}\right)\right)}^{2}=1-\int_{B\left(y, \frac{\bar{r}_{1}}{\sqrt{\beta}}+\varepsilon_{\beta}\right)} e_{1}^{2} d x+\int_{A\left(y, \frac{\bar{r}_{1}}{\sqrt{\beta}}, \frac{\bar{r}_{1}}{\sqrt{\beta}}+\varepsilon_{\beta}\right)} \tilde{u}_{\beta, y}^{2} d x
$$

and

$$
\int_{\Omega \backslash \bar{B}\left(y, \frac{\bar{r}_{1}}{\sqrt{\beta}}\right)}\left|D \tilde{u}_{\beta, y}\right|^{2} d x=\lambda_{1}-\int_{B\left(y, \frac{\bar{r}_{1}}{\sqrt{\beta}}+\varepsilon_{\beta}\right)}\left|D e_{1}\right|^{2} d x+\int_{A\left(y, \frac{\bar{r}_{1}}{\sqrt{\beta}}, \frac{\bar{r}_{1}}{\sqrt{\beta}}+\varepsilon_{\beta}\right)}\left|D \tilde{u}_{\beta, y}\right|^{2} d x .
$$

Since $\lim _{\beta \rightarrow+\infty} \varepsilon_{\beta} \sqrt{\beta}=+\infty$, one can easily verify that there exists a positive number $c(y)$ (depending only on $y$ ) such that

$$
\lim _{\beta \rightarrow+\infty} \varepsilon_{\beta}^{-N} \int_{B\left(y, \frac{\bar{r}_{1}}{\sqrt{\beta}}+\varepsilon_{\beta}\right)}\left(e_{1}^{2}+\left|D e_{1}\right|^{2}\right) d x \leq c(y)
$$

and

$$
\limsup _{\beta \rightarrow+\infty} \varepsilon_{\beta}^{-N} \int_{A\left(y, \frac{\bar{r}_{1}}{\sqrt{\beta}}, \frac{\bar{r}_{1}}{\sqrt{\beta}}+\varepsilon_{\beta}\right)} \tilde{u}_{\beta, y}^{2} d x \leq c(y) .
$$

Now, let us estimate the integral $\int_{A\left(y, \frac{\bar{r}_{1}}{\sqrt{\beta}}, \frac{\bar{r}_{1}}{\sqrt{\beta}}+\varepsilon_{\beta}\right)}\left|D \tilde{u}_{\beta, y}\right|^{2} d x$. Let us write $\tilde{u}_{\beta, y}$ as $\tilde{u}_{\beta, y}=$ $\tilde{v}_{\beta, y}+\tilde{w}_{\beta, y}$ where $\tilde{v}_{\beta, y}$ and $\tilde{w}_{\beta, y}$ satisfy $\Delta \tilde{v}_{\beta, y}=0, \Delta \tilde{w}_{\beta, y}=0$ in $A\left(y, \frac{\bar{r}_{1}}{\sqrt{\beta}}, \frac{\bar{r}_{1}}{\sqrt{\beta}}+\varepsilon_{\beta}\right)$ with boundary condition $\tilde{v}_{\beta, y}=e_{1}, \tilde{w}_{\beta, y}=-e_{1}$ on $\partial B\left(y, \frac{\bar{r}_{1}}{\sqrt{\beta}}\right)$ and $\tilde{v}_{\beta, y}=0, \tilde{w}_{\beta, y}=-e_{1}$ on $\partial B\left(y, \frac{\bar{r}_{1}}{\sqrt{\beta}}+\varepsilon_{\beta}\right)$.

If $N \geq 3$, one can verify by standard arguments that

$$
\lim _{\beta \rightarrow+\infty} \beta^{\frac{N-2}{2}} \int_{A\left(y, \frac{\bar{r}_{1}}{\sqrt{\beta}}, \frac{\bar{r}_{1}}{\sqrt{\beta}}+\varepsilon_{\beta}\right)}\left|D \tilde{v}_{\beta, y}\right|^{2} d x=e_{1}^{2}(y) \int_{\mathbb{R}^{N} \backslash \bar{B}\left(0, \bar{r}_{1}\right)}|D \tilde{V}|^{2} d x=e_{1}^{2}(y) \operatorname{cap}\left(\bar{r}_{1}\right),
$$


where $\tilde{V}$ satisfies $\Delta \tilde{V}=0$ in $\mathbb{R}^{N} \backslash \bar{B}\left(0, \bar{r}_{1}\right), \tilde{V}=1$ on $\partial B\left(0, \bar{r}_{1}\right), \lim _{|x| \rightarrow \infty} \tilde{V}(x)=0$. Moreover,

$$
\limsup _{\beta \rightarrow+\infty} \varepsilon_{\beta}^{-N} \int_{A\left(y, \frac{\bar{n}_{1}}{\sqrt{\beta}}, \frac{\bar{n}_{1}}{\sqrt{\beta}}+\varepsilon_{\beta}\right)}\left|D \tilde{w}_{\beta, y}\right|^{2} d x \leq c(y),
$$

where $c(y)$ is the positive number appearing in (3.11) and (3.12). Taking into account the choice of $\varepsilon_{\beta}$, we obtain also

$$
\lim _{\beta \rightarrow+\infty} \beta^{\frac{N-2}{2}} \int_{A\left(y, \frac{\bar{r}_{1}}{\sqrt{\beta}}, \frac{\bar{r}_{1}}{\sqrt{\beta}}+\varepsilon_{\beta}\right)}\left|D \tilde{v}_{\beta, y}\right|\left|D \tilde{w}_{\beta, y}\right| d x=0 .
$$

Thus, since $\alpha_{\beta} \leq \int_{\Omega}\left|D \bar{u}_{\beta, y}^{-}\right|^{2} d x$ for $\beta>0$ large enough so that $B\left(y, \frac{\bar{r}_{1}}{\sqrt{\beta}}+\varepsilon_{\beta}\right) \subset \Omega$, it follows that

$$
\limsup _{\beta \rightarrow+\infty} \beta^{\frac{N-2}{2}}\left(\alpha_{\beta}-\lambda_{1}\right) \leq e_{1}^{2}(y) \operatorname{cap}\left(\bar{r}_{1}\right) \quad \forall y \in \Omega,
$$

which (as $y$ tends to the boundary of $\Omega$ ) implies (3.6).

If $N=2$, we argue in analogous way (but with $\lg \beta$ in place of $\beta^{\frac{N-2}{2}}$ ). Since $\varepsilon_{\beta}=\frac{1}{\lg \beta}$ for $N=2$, one can verify by direct computation that

$$
\lim _{\beta \rightarrow+\infty} \lg \beta \int_{A\left(y, \frac{\bar{r}_{1}}{\sqrt{\beta}}, \frac{\bar{r}_{1}}{\sqrt{\beta}}+\varepsilon_{\beta}\right)}\left|D \tilde{v}_{\beta, y}\right|^{2} d x=4 \pi e_{1}^{2}(y) .
$$

Moreover,

$$
\limsup _{\beta \rightarrow+\infty} \lg ^{2} \beta \int_{A\left(y, \frac{\bar{r}_{1}}{\sqrt{\beta}}, \frac{\bar{r}_{1}}{\sqrt{\beta}}+\varepsilon_{\beta}\right)}\left|D \tilde{w}_{\beta, y}\right|^{2} d x \leq c(y)
$$

(where $c(y)$ is the same as in (3.11) and (3.12)) and

$$
\lim _{\beta \rightarrow+\infty} \lg \beta \int_{A\left(y, \frac{\bar{r}_{1}}{\sqrt{\beta}}, \frac{\bar{r}_{1}}{\sqrt{\beta}}+\varepsilon_{\beta}\right)}\left|D \tilde{v}_{\beta, y}\right|\left|D \tilde{w}_{\beta, y}\right| d x=0 .
$$

Therefore, it follows that

$$
\limsup _{\beta \rightarrow+\infty} \lg \beta\left(\alpha_{\beta}-\lambda_{1}\right) \leq 4 \pi e_{1}^{2}(y) \quad \forall y \in \Omega
$$

which clearly implies (3.7).

q.e.d.

As a consequence of Propositions 3.2 and 3.3 we can state the following corollary.

Corollary 3.4 For every positive integer $k$, there exists $\tilde{b}_{k}>0$ such that $\alpha_{\beta}<\alpha_{k, \beta}$ $\forall \beta>\tilde{b}_{k}$. 
The proof follows directly by comparing formulas (3.4) and (3.5) with (3.6) and (3.7).

Remark 3.5 The proof of Proposition 3.3 suggests that, for $\beta>0$ large enough, the support of $u_{\beta}^{+}$is localized near the boundary of $\Omega$. Indeed, a more careful analysis of the asymptotic behaviour of $u_{\beta}$ as $\beta \rightarrow+\infty$ (arguing as in [37]) shows that for all $\beta>0$ there exists $y_{\beta} \in \Omega$ such that (up to a subsequence) $y_{\beta}$ converges, as $\beta \rightarrow+\infty$, to a point $\bar{y} \in \partial \Omega$ and the rescaled function $\left(\sup _{\Omega} u_{\beta}\right)^{-1} u_{\beta}\left(y_{\beta}+\frac{x}{\sqrt{\beta}}\right)$ converges to a function $\bar{U}$ such that

$$
\Delta \bar{U}+\bar{U}^{+}=0, \quad \bar{U}^{+} \not \equiv 0, \bar{U}^{-} \not \equiv 0 \quad \text { in } H, \quad \bar{U}=0 \quad \text { on } \partial H
$$

with $H=\left\{x \in \mathbb{R}^{N}:(x \cdot \bar{\nu})<0\right\}$, where $\bar{\nu}$ denotes the outward normal to $\partial \Omega$ in $\bar{y}$. Moreover (arguing as in [37]) for $\beta$ large enough one can construct multibumps eigenfunctions for the Fučík spectrum, having an arbitrarily large number of bumps localized near prescribed connected components of $\partial \Omega$.

In fact, if $\Omega$ is a smooth bounded connected domain of $\mathbb{R}^{N}$ with $N \geq 2$, arguing as in [37], $\forall k \in \mathbb{N}$ one can construct, for $\beta>0$ large enough, a $k$-bumps eigenfunction $\bar{u}_{k, \beta}$, corresponding to a pair $\left(\bar{\alpha}_{k, \beta}, \beta\right) \in \Sigma$, with $k$ bumps localized near $\partial \Omega$ and having asymptotic profile described by the functions $\bar{U}$. Here $\bar{\alpha}_{k, \beta}$ depends continuously on $\beta$ and, for $\beta>0$ large enough, we have $\bar{\alpha}_{k, \beta}<\bar{\alpha}_{k+1, \beta} \forall k \in \mathbb{N}$ and $\bar{\alpha}_{k_{1}, \beta}<\alpha_{k_{2}, \beta}$ $\forall k_{1}, k_{2} \in \mathbb{N}$. Thus, if $N \geq 2$, we obtain a new class of infinitely many curves in $\Sigma$, asymptotic to the lines $\left\{\lambda_{1}\right\} \times \mathbb{R}$ and $\mathbb{R} \times\left\{\lambda_{1}\right\}$, while in the case $N=1$ there exist only two curves having this property.

Remark 3.6 Notice that the difference between the cases $N=1$ and $N \geq 2$ is even more evident if we replace the Dirichlet boundary condition by the Neumann condition $\frac{\partial u}{\partial \nu}=0$ on $\partial \Omega$. In fact, if we denote by $\tilde{\lambda}_{1}<\tilde{\lambda}_{2} \leq \tilde{\lambda}_{3} \leq \ldots$ and by $\widetilde{\Sigma}$, respectively, the eigenvalues of the Laplace operator $-\Delta$ and the Fučík spectrum with Neumann boundary conditions, we have $\tilde{\lambda}_{1}=0$ and, if $N=1$, no curve of $\widetilde{\Sigma}$ is asymptotic to the lines $\{0\} \times \mathbb{R}$ or $\mathbb{R} \times\{0\}$. Indeed, a direct computation shows that, for $N=1$, every nontrivial pair $(\alpha, \beta)$ of $\widetilde{\Sigma}$ satisfies $\alpha>\frac{1}{4} \tilde{\lambda}_{2}$ and $\beta>\frac{1}{4} \tilde{\lambda}_{2}$, with $\tilde{\lambda}_{2}>0$. On the contrary, in the case $N \geq 2$ there exist infinitely many curves contained in $\widetilde{\Sigma}$ and asymptotic to the lines $\{0\} \times \mathbb{R}$ or $\mathbb{R} \times\{0\}$. The corresponding eigenfunctions have an arbitrarily large number of bumps localized in the interior of $\Omega$ or near prescribed connected components of $\partial \Omega$. Both, interior and boundary bumps, present the same asymptotic profile, described by the function $U$ introduced in Proposition 3.2 (while, in case of Dirichlet boundary conditions, the asymptotic profile is described by the function $U$ for the interior bumps and by the function $\bar{U}$ for the boundary bumps).

Notice that, as pointed out in $[3,15,20]$, in case of Neumann boundary conditions there exists a strict connection between the nonexistence of curves in $\widetilde{\Sigma}$, asymptotic to the lines $\{0\} \times \mathbb{R}$ and $\mathbb{R} \times\{0\}$, and the fact that the antimaximum principle (see [14]) holds uniformly (in a suitable sense). 
Remark 3.7 For the sake of simplicity, in this paper we have considered only the case of the Laplace operator, but the results we have presented may be easily extended to cover the case of more general boundary conditions and elliptic operators in divergence form. Moreover, the variational method we have used in this paper may be easily adapted to deal also with quasilinear operators as the $p$-laplacian. Thus, we can obtain also for the $p$-laplacian a variational characterization of the first nontrivial curve of the Fučík spectrum, similar to Theorem 2.1 (but the asymptotic behaviour depends on $p$, on the spatial dimension $N$ and on the boundary conditions we consider).

Acknowledgement. The authors have been supported by the "Gruppo Nazionale per l'Analisi Matematica, la Probabilità e le loro Applicazioni (GNAMPA)" of the Istituto Nazionale di Alta Matematica (INdAM).

\section{References}

[1] A. Ambrosetti, G. Prodi, On the inversion of some differentiable mappings with singularities between Banach spaces. Ann. Mat. Pura Appl. (4) 93 (1972), 231-246.

[2] M. Arias, J. Campos, Radial Fučík spectrum of the Laplace operator. J. Math. Anal. Appl. 190 (3) (1995), 654-666.

[3] M. Arias, J. Campos, J.-P. Gossez, On the antimaximum principle and the Fučík spectrum for the Neumann $p$-Laplacian. Differential Integral Equations 13 (1-3) (2000), $217-$ 226 .

[4] V. Benci, A geometrical index for the group $S^{1}$ and some applications to the study of periodic solutions of ordinary differential equations. Comm. Pure Appl. Math. 34 (1981), no. $4,393-432$.

[5] A.K. Ben-Naoum, C. Fabry, D. Smets, Structure of the Fučík spectrum and existence of solutions for equations with asymmetric nonlinearities. Proc. Roy. Soc. Edinburgh Sect. A 131 (2001), no. 2, 241-265.

[6] H. Berestycki, Le nombre de solutions de certains problémes semi-linéaires elliptiques. J. Funct. Anal. 40 (1981), no. 1, 1-29.

[7] H. Berestycki, J.-M. Lasry, G. Mancini, B. Ruf, Existence of multiple periodic orbits on star-shaped Hamiltonian surfaces. Comm. Pure Appl. Math. 38 (1985), no. 3, 253-289.

[8] N.P. Các, On nontrivial solutions of a Dirichlet problem whose jumping nonlinearity crosses a multiple eigenvalue. J. Differential Equations 80 (1989), no. 2, 379-404.

[9] N.P. Các, On a boundary value problem with nonsmooth jumping nonlinearity. J. Differential Equations 93 (1991), no. 2, 238-259. 
[10] R. Caccioppoli, Un principio di inversione per le corrispondenze funzionali e sue applicazioni alle equazioni alle derivate parziali. Atti Acc. Naz. Lincei 16 (1932), 392-400.

[11] G. Cerami, R. Molle, D. Passaseo, Multiplicity of positive and nodal solutions for scalar field equations. J. Differential Equations (2014), http://dx.doi.org/10.1016/ j.jde.2014.07.002.

[12] G. Cerami, D. Passaseo, S. Solimini, Infinitely many positive solutions to some scalar field equations with nonsymmetric coefficients. Comm. Pure Appl. Math. 66 (2013), no. $3,372-413$.

[13] G. Cerami, D. Passaseo, S. Solimini, Nonlinear scalar field equations: existence of a solution with infinitely many bumps. Ann. I. H. Poincaré - AN (2013), http://dx.doi.org/ 10.1016/j.anihpc.2013.08.008.

[14] P. Clement, L. Peletier, An antimaximum principle for second order elliptic operators. J. Diff. Eq., 34 (1979), 218-229.

[15] M. Cuesta, D. de Figueiredo, J.-P. Gossez, The beginning of the Fučík spectrum for the p-Laplacian. J. Differential Equations 159 (1) (1999), 212-238.

[16] M. Cuesta, J.-P. Gossez, A variational approach to nonresonance with respect to the Fučík spectrum. Nonlinear Anal. 19 (1992), no. 5, 487-500.

[17] E.N. Dancer, On the Dirichlet problem for weakly non-linear elliptic partial differential equations. Proc. Roy. Soc. Edinburgh Sect. A 76, (1976/77), no. 4, 283-300.

[18] E.N. Dancer, On the existence of solutions of certain asymptotically homogeneous problems. Math. Z. 177 (1981), no. 1, 33-48.

[19] E.N. Dancer, Generic domain dependence for nonsmooth equations and the open set problem for jumping nonlinearities. Topol. Methods Nonlinear Anal. 1 (1993), no. 1, $139-150$.

[20] D.G. de Figueiredo, J.-P. Gossez, On the first curve of the Fučík spectrum of an elliptic operator. Differential Integral Equations 7 (1994), no. 5-6, 1285-1302.

[21] S. Fučík, Nonlinear equations with noninvertible linear part. Czechoslovak Math. J. 24(99) (1974), 467-495.

[22] S. Fučík, Boundary value problems with jumping nonlinearities. Časopis Pěst. Mat. 101 (1976), no. 1, 69-87.

[23] S. Fučík, A. Kufner, Nonlinear differential equations. Studies in Applied Mechanics, 2. Elsevier Scientific Publishing Co., Amsterdam-New York, 1980. 
[24] T. Gallouët, O. Kavian, Résultats d'existence et de non-existence pour certains problèmes demi-linéaires à l'infini. Ann. Fac. Sci. Toulouse Math. (5) 3 (1981), no. 3-4, $201-246$

[25] T. Gallouët, O. Kavian, Resonance for jumping nonlinearities. Comm. Partial Differential Equations 7 (1982), no. 3, 325-342.

[26] J.V.A. Gonçalves, C.A. Magalhães, Semilinear elliptic problems with crossing of the singular set. Trabalhos de Matemática n. 263, Univ. de Brasilia (1992).

[27] J. Horák, W. Reichel, Analytical and numerical results for the Fučík spectrum of the Laplacian. J. Comput. Appl. Math. 161 (2003), no. 2, 313-338.

[28] C. Li, S. Li, Z. Liu, J. Pan, On the Fučík spectrum. J. Differential Equations 244 (2008), no. $10,2498-2528$.

[29] C.A. Magalhães, Semilinear elliptic problem with crossing of multiple eigenvalues. Comm. Partial Differential Equations 15 (1990), no. 9, 1265-1292.

[30] C.A. Margulies, W. Margulies, An example of the Fučík spectrum. Nonlinear Anal. 29 (1997), no. 12, 1373-1378.

[31] W. Marzantowicz, A Borsuk-Ulam theorem for orthogonal $T^{k}$ and $Z_{p}^{r}$ actions and applications. J. Math. Anal. Appl. 137 (1989), no. 1, 99-121

[32] E. Massa, B. Ruf, On the Fučík spectrum of the Laplacian on a torus. J. Funct. Anal. 256 (2009), no. 5, 1432-1452.

[33] R. Molle, D. Passaseo, Multiple solutions for a class of elliptic equations with jumping nonlinearities. Ann. Inst. H. Poincaré Anal. Non Linéaire (2010), no. 2, 529-553.

[34] R. Molle, D. Passaseo, Existence and multiplicity of solutions for elliptic equations with jumping nonlinearities. J. Funct. Anal. 259 (2010), no. 9, 2253-2295.

[35] R. Molle, D. Passaseo, Elliptic equations with jumping nonlinearities involving high eigenvalues. Calc. Var. Partial Differential Equations, 49 (2014), no. 1/2, 861-907.

[36] R. Molle, D. Passaseo, New properties of the Fučík spectrum. C. R. Math. Acad. Sci. Paris 351 (2013), no. 17/18, 681-685.

[37] R. Molle, D. Passaseo, Infinitely many new curves of the Fučík spectrum. Ann. I. H. Poincaré - AN (2014), http://dx.doi.org/10.1016/j.anihpc.2014.05.007.

[38] R. Molle, D. Passaseo, On the first curve of the Fučík spectrum for elliptic operators. Atti Accad. Naz. Lincei Rend. Lincei Mat. Appl. 25 (2014), no. 2, 141-146.

[39] P.H. Rabinowitz, Minimax methods in critical point theory with applications to differential equations. CBMS Regional Conference Series in Mathematics, 65. American Mathematical Society, Providence, RI, 1986. 
[40] B. Ruf, On nonlinear elliptic problems with jumping nonlinearities. Ann. Mat. Pura Appl. (4) 128 (1981), 133-151.

[41] M. Schechter, The Fučík spectrum. Indiana Univ. Math. J. 43 (1994), no. 4, 1139-1157.

[42] M. Schechter, Type (II) regions between curves of the Fucik spectrum. Nonlinear Differential Equations Appl. 4 (1997), no. 4, 459-476.

[43] S. Solimini, Some remarks on the number of solutions of some nonlinear elliptic problems. Ann. Inst. H. Poincaré - Anal. Non Linéaire 2 (1985), no. 2, 143-156. 